\title{
PEAK3 pseudokinase represents a pro-migratory and -invasive signalling scaffold
}

Jianmei Hou ${ }^{1,2}$, Elizabeth $\vee$ Nguyen ${ }^{1,2}$, Minglyanna Surudoi ${ }^{3,4}$, Michael $\mathrm{J}$ Roy ${ }^{3,4}$, Onisha Patel ${ }^{3,4}$, Isabelle S Lucet ${ }^{3,4}$, Xiuquan Ma ${ }^{1,2,5}$ and Roger J Daly ${ }^{1,2,5,6}$

1 Cancer Program, Biomedicine Discovery Institute, Monash University, Melbourne, VIC, 3800, Australia.

2 Department of Biochemistry and Molecular Biology, Monash University, Melbourne, VIC, 3800, Australia.

3 The Walter and Eliza Hall Institute of Medical Research, Parkville, VIC, 3052, Australia.

4 Department of Medical Biology, University of Melbourne, Parkville, VIC, 3052, Australia.

5 Co-senior authors.

6 To whom correspondence should be addressed: roger.daly@monash.edu

Key words: SgK269, SgK223, Pragmin, signal transduction 


\begin{abstract}
The PEAK family of pseudokinases comprises PEAK1 and PEAK2 as well as the recently-identified PEAK3. PEAK1/2 play fundamental roles in regulating tyrosine kinase signal output and oncogenesis, while PEAK3 remains poorly-characterized. Here, we demonstrate that PEAK3 undergoes homotypic association as well as heterotypic interaction with PEAK1/2. PEAK3 also recruits ASAP1/2, Cbl and PYK2 and the adaptors Grb2 and CrkIl, with binding dependent on PEAK3 dimerization. PEAK3 tyrosine phosphorylation on Y24 is also dependent on dimerization as well as Src family kinase activity, and interestingly, is decreased via PTPN12 in response to EGF treatment. Y24 phosphorylation is required for binding of Grb2 and ASAP1. Overexpression of PEAK3 in MDA-MB231 breast cancer cells enhanced cell elongation and cell motility, while knockdown of endogenous PEAK3 decreased cell migration. In addition, overexpression of PEAK3 in PEAK1/2 compound knockout MCF-10A breast epithelial cells enhanced acinar growth and invasion in 3D culture, with the latter phenotype dependent on PEAK3 tyrosine phosphorylation and binding of Grb2 and ASAP1. These findings characterize PEAK3 as an integral member of the PEAK family with scaffolding roles that promote cell proliferation, migration and invasion.
\end{abstract}

\title{
Introduction
}

Approximately $10 \%$ of human protein kinases are classified as pseudokinases since they lack at least one of the conserved amino acid motifs important for catalytic function (Manning et al, 2002; Reiterer et al, 2014). Although most of them are predicted to be catalytically inactive they play important signalling roles by functioning as scaffolds, anchors, or allosteric regulators (Reiterer et al., 2014). Three pseudokinases, PEAK1 (also known as SgK269), PEAK2 (SgK223/Pragmin) and the recentlyidentified PEAK3 (Chromosome 19 Open Reading Frame 35, C19orf35) form the PEAK family. These three proteins share highly conserved domain structures including a C-terminal pseudokinase (PsK) domain with adjacent $\mathrm{N}$ - and C-terminal a-helical regions (Patel et al, 2020). In addition, they all contain specific N-terminal extensions, which are long in PEAK1 ( 1,200 residues) and PEAK2 ( 900 residues), but much shorter in PEAK3 ( $\sim 100$ residues) (Patel et al., 2020).

Both PEAK1 and PEAK2 function as scaffolds, harbouring tyrosine phosphorylation sites that specifically recruit src homology $(\mathrm{SH}) 2$ and phosphotyrosine binding (PTB) domain-containing effectors to regulate cell proliferation and/or migration/invasion. PEAK1 is phosphorylated by activated Src family kinases (SFKs) downstream of the epidermal growth factor receptor (EGFR) and specific integrins and regulates the p130Cas-Crk-paxillin, Erk and Shc1 signaling pathways to promote cell migration and invasion (Croucher et al, 2013; Wang et al, 2010; Zheng et al, 2013). Phosphorylation of PEAK1 Y635 creates a binding site for the Grb2 SH2 domain and leads to Ras signalling pathway activation and aberrant growth of MCF-10A mammary epithelial cells in 3D (Croucher et al., 2013). Also, binding of PEAK1 Y1188 to the Shc1 PTB domain enables Shc1 to switch from regulating Grb2dependent mitogenic activity to PEAK1-orchestrated cell morphology and invasion (Zheng et al., 2013). Another important function of PEAK1 is that it represents a regulator of epithelial-mesenchymal transition (EMT) (Croucher et al., 2013). For example, in MCF-10A mammary epithelial cells, overexpression of PEAK1 results in these cells converting to an elongated, mesenchymal 
morphology, and stable knockdown of PEAK1 in MDA-MB-231 breast cancer cells leads to mesenchymal-epithelial transition (MET) (Croucher et al., 2013). Several studies over the last decade have reported PEAK1 overexpression in multiple human malignancies including breast (Agajanian et al, 2015; Croucher et al., 2013), colon (Huang et al, 2018; Wang et al., 2010), lung (Ding et al, 2018) and pancreatic cancers (Kelber et al, 2012), suggesting an oncogenic role in cancer growth and progression. In the case of PEAK2, the EPIY ${ }_{411} \mathrm{~A}$ motif serves as a docking site for the $\mathrm{SH} 2$ domain of the C-terminal Src kinase (Csk), highlighting a critical role for PEAK2 in controlling Csk localisation to regulate SFK activity (Safari et al, 2011). Similar to PEAK1, PEAK2 induces cell elongation and co-expression of Csk and PEAK2 markedly induces cell scattering (Senda et al, 2016). PEAK2 is required for the invasion of colon carcinoma cells (Leroy et al, 2009) and PEAK2 expression is significantly increased in pancreatic cancer and associated with poor prognosis in non-small cell lung cancer (Kong et al, 2016; Tactacan et al, 2015).

PEAK1 and PEAK2 form homo- and hetero-dimers, this representing an important regulatory mechanism in their downstream signaling and function (Ha \& Boggon, 2018; Liu et al, 2016; Patel et al, 2017). Homo- and heterotypic association of PEAK1 and PEAK2 is mediated by the highlyconserved split helical dimerization (SHED) domain, comprised of the helix immediately $\mathrm{N}$-terminal to the PsK domain and 3 helices C-terminal to the PsK domain that form an "XL"-shaped helical bundle (Ha \& Boggon, 2018; Liu et al., 2016; Patel et al., 2017). The ability of PEAK1 to enhance cell elongation and migration is dependent on the functionality of the SHED region. In addition, PEAK1 requires PEAK2 to efficiently promote cell migration and activate Stat3 in MCF-10A cells and PEAK1 bridges PEAK2 to Grb2 (Liu et al., 2016). Therefore, homo- and heterotypic association of these scaffolds is critical to biological activity and acts as a mechanism to diversify signalling outputs (Liu et al., 2016).

The third family member, C19orf35 or PEAK3, was identified by the Roche group in 2018 (Lecointre et al, 2018), although its signalling mechanism and function remain unclear. Recently, it was demonstrated via site-directed mutagenesis and co-immunoprecipitation studies that PEAK3 also homo-dimerizes via the highly conserved SHED domain, this being critical for Crkll recruitment (Lopez et al, 2019). In addition, while PEAK3 overexpression did not impact cell morphology, it prevented Crkll-dependent membrane ruffling, leading the authors to suggest that PEAK3 may represent a negative modulator of PEAK1/2 action (Lopez et al., 2019).

In this study, we characterize PEAK3 signalling mechanisms and function in detail. The resulting data indicate that PEAK3 has a scaffolding function, a pro-migratory and -invasive role, and demonstrate important functional roles for tyrosine phosphorylation, dimerization and recruitment of specific effectors including Grb2 and ASAP1.

\section{Results}

\section{PEAK3 undergoes heterotypic association with PEAK1 and PEAK2}

PEAK3 exhibits a similar molecular structure to PEAK1 and PEAK2 (Fig 1A). They all share a conserved C-terminal PsK domain with adjacent $\mathrm{N}$ - and C-terminal a-helical regions forming the 
highly-conserved split helical dimerization (SHED) domain, and a N-terminal extension which is significantly shorter in PEAK3. PEAK1 and PEAK2 harbour a Crkll SH3 domain binding motif (PPPLPKK) which is conserved in PEAK3 (residues 56-62) (Lopez et al., 2019). Additionally, PEAK3 contains a SH2 binding motif (YSNL, residues 24-27) at the N-terminus and another potential SH3 binding motif (PGAPWR, residues 243-248), located in the PsK domain (Fig 1A).

The presence of the conserved SHED domain in PEAK3 and demonstration that it is critical for PEAK3 homotypic association (Lopez et al., 2019), led us to determine whether PEAK3 undergoes heterotypic association with PEAK1 and/or PEAK2. This was undertaken by expressing HA-tagged PEAK3 in parental MCF-10A cells. In addition, we performed similar studies in PEAK1 or PEAK2 knock-out (KO) MCF-10A cells generated by CRISPR (Fig EV1A) (Liu et al., 2016; Patel et al., 2017) in order to rule out 'bridging' of PEAK3 binding to PEAK1 by PEAK2 (and vice versa), and utilized PEAK3 SHED mutations predicted to disrupt dimerization (Fig 1B) (Lopez et al., 2019) in order to confirm the underlying association mechanism. PEAK3 associated with both PEAK1 and PEAK2 in parental MCF-10A cells and interacted with PEAK1 in the absence of PEAK2, and PEAK2 in the absence of PEAK1 (Fig 1C). These data indicate that PEAK3 can form heterotypic complexes with PEAK1 or PEAK2. Furthermore, introduction of SHED mutations abolished the ability of PEAK3 to associate with PEAK1 and PEAK2 (Figs 1C and EV1B). These data indicate that PEAK3 undergoes heterotypic association with PEAK1 and PEAK2 via the conserved dimerization interface.

\section{Characterization of the PEAK3 scaffolding function and its regulation by EGF}

The existence of $\mathrm{SH} 2$ and $\mathrm{SH} 3$ binding motifs (Fig 1A) indicates that PEAK3 may function as a scaffold, assembling protein complexes by recruiting specific binding partners. To address this hypothesis, we undertook LC-MS/MS analysis of anti-HA immunoprecipitates (IPs) prepared from MCF-10A cells expressing HA-tagged PEAK3. Proteins exhibiting significantly increased abundance in PEAK3 IPs versus control IPs were identified (Table EV1) and are presented in a volcano plot (Fig 2A, left panel). This confirmed PEAK3 interaction with Crkll (Lopez et al., 2019), independently verified PEAK1 binding (Fig 1C) and also revealed a variety of other binding partners including the adaptors CrkL and Grb2, the ADP-ribosylation factor (ARF) GTPase-activating proteins ASAP1 and ASAP2, and non-receptor protein tyrosine kinase PYK2. In addition, since growth factor stimulation markedly impacts the assembly of PEAK1 signalling complexes (Zheng et al., 2013), the effect of EGF treatment on the PEAK3 interactome was also characterized (Fig 2A, right panel and Table EV1). Interestingly, this revealed that the ability of PEAK3 to associate with ASAP1/2 and Grb2 was decreased by 5 min EGF treatment, whereas recruitment of the E3 ubiquitin ligase $\mathrm{Cbl}$ was increased.

To complement these analyses, we undertook anti-phosphotyrosine Western blotting of anti-HA IPS prepared from control or EGF-treated PEAK1/2 double KO (DKO) MCF-10A cells (Fig EV1A) expressing HA-tagged PEAK3 (Fig 2B). This detected a $\sim 125 \mathrm{kDa}$ band that was decreased by EGF treatment, and a $\sim 50 \mathrm{kDa}$ band that increased. Based on size, the defined interactome (Fig 2A) and known PEAK1 binding partners, possible candidates represent ASAP1 and either PEAK3 or Shc1, respectively. In addition, another two bands at $\sim 175$ and $\sim 120 \mathrm{kDa}$ were detected at $5 \mathrm{~min}$ and $20 \mathrm{~min}$ after EGF treatment, which may correspond to the EGFR and Cbl, respectively. Consistent with the 
IP/MS and anti-phosphotyrosine blotting results, IP/Westerns with specific antibodies demonstrated association of PEAK3 with Grb2, Crkll, PYK2, ASAP1 and EGFR under control conditions, and EGF treatment led to decreased association of PEAK3 with Grb2 and ASAP1 within 5 min, and increased interaction with the EGFR and Cbl, peaking after 20 min stimulation (Fig 2C). Of note, binding of Crkll was also rapidly reduced following EGF stimulation, but this returned to control levels after $20 \mathrm{~min}-3$ h EGF treatment (Fig 2C).

In order to resolve the identity of the $50 \mathrm{kDa}$ tyrosine phosphorylated protein detected in PEAK3 IPS (Fig 2B), we first denatured the lysates prior to IP, so that the IP would only isolate PEAK3 and not associated proteins. Unexpectedly, this revealed that tyrosine phosphorylation of PEAK3 was downregulated by EGF treatment (Fig 3A), indicating that one or more other PEAK3 interactor(s) must contribute to the upregulated $\sim 50 \mathrm{kDa}$ pY band. A top candidate was Shc1, a well-characterized PEAK1 and Grb2 binding partner (Zheng et al., 2013). Indeed, direct blotting revealed that association of both total and Y317-phosphorylated Shc1 with PEAK3 was increased by EGF treatment, reaching a maximum at $5 \mathrm{~min}$ and then decreasing (Fig 2C). The dynamics of the PEAK3 interactome in response to EGF treatment are summarized in Fig EV2. Collectively, these data indicate that PEAK3 functions as a scaffold and via specific downstream effectors may regulate a variety of processes and biological endpoints including cytoskeletal dynamics and cell motility, EGF downstream signalling and protein ubiquitylation and turnover.

\section{Regulation of PEAK3 tyrosine phosphorylation}

Our finding that PEAK3 is rapidly dephosphorylated in response to EGF stimulation (Fig 3A and D) led us to interrogate the underlying mechanism. It was previously established that site-selective tyrosine phosphorylation of Shc1 is temporally regulated following EGF stimulation, with the protein tyrosine phosphatase (PTP) N12 dephosphorylating the Shc1/Grb2 complex prior to recruitment of PEAK1 to Shc1 to promote cell migration/invasion (Zheng et al., 2013). This linkage of PTPN12 to PEAK family signalling led us to test its role in regulating PEAK3 tyrosine phosphorylation. Indeed, knockdown of PTPN12 in MCF-10A DKO cells enhanced basal phosphorylation of PEAK3 and markedly attenuated EGF-induced dephosphorylation of this scaffold, demonstrating that the latter process is PTPN12-dependent (Fig 3A). In addition, since tyrosine phosphorylation of PEAK1 is mediated by SFKs (Croucher et al., 2013; Wang et al., 2010), we characterized the role of these kinases. Treatment of MCF-10A DKO cells with either the selective SFK inhibitor eCF506 (Fraser et al, 2016) or the SFK/Abl inhibitor dasatinib blocked PEAK3 tyrosine phosphorylation, indicating that this modification is dependent on SFK activity (Fig 3B).

In order to determine the role of dimerization and specific $\mathrm{SH} 2$ and $\mathrm{SH} 3$ binding motifs in PEAK3 tyrosine phosphorylation, HA-tagged PEAK3 proteins with mutations in the dimerization interface and specific SH2 and SH3 binding motifs (Figs $1 \mathrm{~B}$ and $3 \mathrm{C}$ ) were expressed in MCF-10A DKO cells. IP/Western blotting analysis using denatured lysates demonstrated that dimerization is required for efficient PEAK3 tyrosine phosphorylation (Fig 3D). Mutation of Y24 also abolished PEAK3 tyrosine phosphorylation (Fig 3D), indicating that this is the main phosphorylated site and $\mathrm{Y}_{24} \mathrm{SNL}$ represents a potential SH2 binding motif that conforms to a Grb2 SH2 binding consensus ( $\mathrm{PYXN}$ ) (Miller et al, 
2008). In addition, mutation of either SH3 binding motif also prevented PEAK3 tyrosine phosphorylation (Fig 3D), highlighting a potential role in recruiting or regulating the upstream tyrosine kinase.

\section{Structural requirements for effector recruitment by PEAK3}

Next, we turned to the PEAK3 interactome, and having demonstrated that PEAK3 dimerization is required for PEAK3 tyrosine phosphorylation (Fig 3D), determined how dimerization potential affects recruitment of specific binding partners. Flag-tagged wildtype (WT) PEAK3 was co-expressed in HEK293 cells with HA-tagged versions of WT PEAK3 or dimerization-defective mutants (Fig 4A). IP/Westerns confirmed the inability of these mutants to undergo homotypic association, and also revealed that recruitment of Grb2, Crkll, ASAP1 and PYK2 to PEAK3 was PEAK3 dimerizationdependent (Fig 4A). However, association of EGFR with these mutants was similar to that for wildtype PEAK3 (Fig 4A). These findings build significantly on the previous report from the Jura laboratory reporting dimerization-dependent Crkll association (Lopez et al., 2019), highlighting a much broader impact of dimerization on assembly of the PEAK3 interactome, and also demonstrate that binding of PEAK3 monomer to certain interactors can occur.

The roles of specific SH2/SH3 binding motifs were then characterized. However, in order to correctly interpret these data, it was first necessary to determine whether mutation of these motifs impacted PEAK3 dimerization. Importantly, while mutation of the $\mathrm{SH} 2$ binding motif at $\mathrm{Y} 24$ and the N-terminal motif SH3(1) had no effect, mutation of SH3(2), which is located in the N-lobe of the PsK domain (Fig

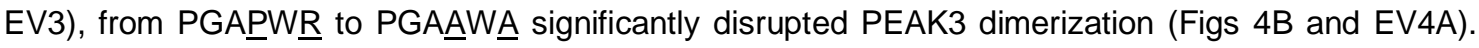
Interestingly, the $\mathrm{SH} 3(2)$ motif is not conserved within the PEAK family and corresponds to the loop between the $\beta 4$ and $\beta 5$ strands that in the PEAK1 and PEAK2 crystal structures adopts an $\alpha$-turn. This region also marks the start of a unique insertion loop in PEAK1 and PEAK2 that is absent in PEAK3. Even though the $\beta 4-\beta 5$ loop is located away from the dimerization domain, the $\mathrm{SH} 3(2)$ mutation may affect the conformation in this region and thereby impact on the orientation of the $\mathrm{N}$ lobe with respect to the C-lobe, the positioning of the $\alpha \mathrm{N} 1$ helix and hence dimerization (Fig EV3).

With regard to recruitment of binding partners, mutation of the PEAK3 SH2 binding motif at Y24 abolished PEAK3 association with Grb2 and ASAP1 and decreased association with Crkll to approximately $40 \%$ of control. However, it had no effect on Cbl, PYK2 and EGFR binding (Figs 4C and EV4B). Disruption of SH3(1) eliminated Crkll and Cbl association, markedly reduced Grb2 and ASAP1 binding, decreased PYK2 recruitment to approximately $50 \%$, and did not affect EGFR association. In the case of $\mathrm{SH} 3(2)$, mutation led to undetectable, or significantly reduced, binding of Grb2, Crkll, ASAP1 PYK2 and Cbl, while association with the EGFR was enhanced (Figs 4C and EV4B). However, additional effects of the $\mathrm{SH} 3$ binding site mutations must be considered when interpreting these data. First, integrity of $\mathrm{SH} 3(1)$ is required for Y24 phosphorylation (Fig 3D). Consequently, mutation of $\mathrm{SH} 3(1)$ will indirectly reduce $\mathrm{Y} 24$ phosphorylation and thereby prevent $\mathrm{SH} 2$ domain-mediated interactions at this site, as well as direct $\mathrm{SH} 3$ domain-mediated binding to $\mathrm{SH} 3(1)$. Second, since mutation of $\mathrm{SH} 3(2)$ negatively impacts both PEAK3 dimerization and tyrosine phosphorylation, this explains the marked reduction in binding partner interactions, although the 
increased association with EGFR may reflect an altered conformation of the PsK domain. Integrating these data with the dynamics of Y24 phosphorylation and Grb2 and ASAP1 binding in response to EGF (Figs 2C and EV2), and considering the modular structure of Grb2 and ASAP1 (Lowenstein et al, 1992; Tanna et al, 2019), it appears likely that Grb2 binds Y24 via its SH2 domain and bridges PEAK3 to ASAP1 (Fig EV4C). The requirement for SH3(1) in ASAP1 binding may reflect its role in indirectly mediating Y24 phosphorylation, binding of SH3(1) to the ASAP1 SH3 domain, or bridging by another adaptor. With regard to the latter mechanism, although the dynamics of ASAP1 recruitment to PEAK3 are closer to those of Grb2 than Crkll (Fig. EV2), it remains possible that Grb2 and Crkll function co-operatively to recruit ASAP1 (Fig EV4D). The mechanisms of recruitment for PYK2, Cbl and EGFR require further characterization, since they do not follow the same kinetics as Grb2 or CrkII binding.

\section{Y24/Grb2/ASAP1 represents a key PEAK3 signalling axis}

Our findings that EGF-induced dissociation of Grb2 and ASAP1 from PEAK3 follow similar kinetics and occurs concurrent with PEAK3 dephosphorylation, and that binding of both proteins to PEAK3 is lost upon Y24 mutation (Figs 2-4), highlight the importance of Y24 phosphorylation to PEAK3 signalling. In order to interrogate this potential signalling axis in more detail, we undertook Isothermal Titration Calorimetry (ITC) experiments to measure the binding of recombinant full-length Grb2 or Crkll to synthetic 7-mer peptides, either tyrosine phosphorylated or non-phosphorylated, encompassing the Y24 SH2 binding motif in PEAK3. This confirmed direct and specific binding of both adaptors to the phosphopeptide with 1:1 stoichiometry, with Grb2 exhibiting a 3-fold higher affinity for this site (measured dissociation constants, $K_{\mathrm{D}}$, of the phosphopeptide were $2.6 \mu \mathrm{M}$ and 7.8 $\mu \mathrm{M}$ for Grb2 and Crkll respectively) (Fig 5A-C), consistent with the greater role for this site in Grb2 recruitment (Fig 4C). To determine the requirement for Grb2 in assembly of the PEAK3 interactome, we knocked down Grb2 then undertook Western blot analysis of PEAK3 IPs. Strikingly, this resulted in loss of ASAP1 binding, while association with PYK2 was unaffected (Fig 5D). However, it also led to undetectable PEAK3 tyrosine phosphorylation and reduced Crkll recruitment (Fig 5D), the latter consistent with the effect of Y24 mutation (Fig 4C). These data indicate that ASAP1 recruitment to PEAK3 is indeed Grb2-dependent, but because PEAK3 tyrosine phosphorylation is also dependent on the presence of Grb2, this may reflect Grb2-dependent Y24 phosphorylation, bridging by Grb2, or both. Nonetheless, they reveal a key PEAK3 signalling axis that can be further characterized in terms of its functional role.

\section{PEAK3 promotes cell elongation and motility}

While PEAK1 and PEAK2 positively regulate cell elongation and migration (Bristow et al, 2013; Croucher et al., 2013; Safari et al., 2011; Tactacan et al., 2015), PEAK3 blocks Crkll-dependent membrane ruffling, suggesting a possible antagonistic role in PEAK family signalling (Lopez et al., 2019). To characterize the functional role of PEAK3, WT PEAK3 or specific PEAK3 mutants were stably expressed in MDA-MB-231 breast cancer cells (Fig 6A). Expression of WT PEAK3 led to clear transition to a more elongated morphology (Fig $6 \mathrm{~B}$ and $\mathrm{C}$ ), but this effect was lost with introduction of the dimerization disrupting mutations $\mathrm{A} 436 \mathrm{E}$ and $\mathrm{SH} 3(2)$ (Fig 6C). 
The role of PEAK3 in regulating cell migration was also characterized. First, we established that overexpression of PEAK3 in MDA-MB-231 cells leads to enhanced random cell motility, as determined by live cell tracking (Fig $6 \mathrm{D}$ and $\mathrm{E}$ ). Next, we characterized the role of endogenous PEAK3. In order to undertake this study, we first generated a custom polyclonal anti-PEAK3 antibody. This detected ectopically-expressed PEAK3 (Fig EV5A) and an approximately 50 kDa band in MDAMB-231 cell lysates that was knocked down by each of 4 independent siRNAs directed against PEAK3 (Fig 6F) and ablated by CRISPR-mediated knockout (Fig EV5B). Endogenous PEAK3 was also detected in RH30 sarcoma and A172 glioblastoma cells (Fig EV5B and C). Having established this assay for PEAK3 protein expression, we characterized the effect of PEAK3 knockdown. Importantly, use of each of the PEAK3-directed siRNAs significantly reduced MDA-MB-231 migration in a scratch assay (Fig 6G), confirming the pro-migratory role of endogenous PEAK3.

\section{PEAK3 promotes growth and invasion of MCF-10A acini in three-dimensional culture}

Growth of MCF-10A breast epithelial cells in 3D Matrigel culture represents a powerful model system for characterizing the effect of specific signalling proteins on a variety of biological endpoints, including proliferation, apoptosis, polarity and invasion (Debnath et al, 2003), and was used to identify PEAK1 as a breast cancer oncogene (Croucher et al., 2013). Therefore, we exploited this model to provide further insights into PEAK3 function. In addition, we utilized DKO MCF-10A cells, in order to interrogate PEAK3 in the absence of PEAK1 and PEAK2. Overexpression of PEAK3 led to a significant increase in acinar size (Fig 7A-C) at Day 5 and Ki67-positive acini at Day 12 (Fig 7D and E), highlighting for the first time a pro-proliferative role for PEAK3 and indicating that expression of this scaffold can overcome the proliferative suppression that normally occurs in late-stage MCF-10A cultures (Debnath et al., 2003). This effect of PEAK3 on acinar growth was reduced for all of the mutants analysed (Fig 7C).

Interestingly, an additional phenotype observed in late stage PEAK3-overexpressing cultures was cell invasion, characterized by a failure in basal membrane integrity leading to cells invading the surrounding matrix (Fig 8A-B). Strikingly, this phenotype was dependent on not only PEAK3 dimerization, but also the two SH3 binding sites and Y24 (Fig 8C). Since the latter is critical for not only Grb2 binding but also recruitment of ASAP1, this highlights an important role for the Y24/Grb2/ASAP1 signalling axis in PEAK3-mediated cell invasion.

\section{Discussion}

The pseudokinases PEAK1 and 2 have emerged as key regulators of cell migration and signalling in both normal and cancer cells (Patel et al., 2020). Based on a shorter N-terminal extension and ability to block Crkll-enhanced membrane ruffling and cell morphology change, it was recently proposed the new family member PEAK3 might represent a negative regulator that antagonizes PEAK $1 / 2$ function (Lopez et al., 2019). However, in this study, through detailed mechanistic and functional characterization, including knockdown of the endogenous protein, we determine that PEAK3 instead represents a positive-acting scaffold that significantly enhances the signalling repertoire and output of the PEAK family and promotes cell proliferation, migration and invasion. In addition, the characterization of PEAK3 reveals complex mechanisms underpinning effector recruitment with 
significant implications for understanding signalling by the PEAK pseudokinases as well as scaffolds and adaptors more generally.

An important finding was that despite its shorter $\mathrm{N}$-terminal extension, PEAK3 recruits several proteins not bound by PEAK1/2, including Cbl, PYK2 and ASAP1. Since we demonstrate that PEAK3 can undergo homo- as well as heterotypic association, these data indicate that PEAK3 has important independent signalling functions and can also expand the signalling potential of the PEAK family by associating with PEAK1/2 and assembling signalling complexes with contrasting outputs. This expansion of signalling repertoire via dimerization is also observed in the erbB family of receptor tyrosine kinases (Citri \& Yarden, 2006), although in this case only one family member is a pseudokinase (erbB3), in contrast to the PEAK family where this is a common feature. With regard to binding of the E3 ubiquitin ligase $\mathrm{Cbl}$ by PEAK3, this might represent a mechanism for promoting ubiquitin-mediated degradation of not only PEAK3 itself but also PEAK1/2 bound as partners in a heterodimer. In addition, since PEAK3 undergoes EGF-induced association with the EGFR, this might also represent a mechanism for promoting EGFR ubiquitylation. Furthermore, given the known roles of the PEAK family in regulating cytoskeletal organization and cell migration/invasion, the identification of PYK2 and ASAP1 as PEAK3 binding partners identifies important downstream effectors that may mediate these effects. In this regard, binding of ASAP1 provides a potential mechanism to promote cell invasion, since ASAP1 enhances podosome and invadopodia formation in transformed cells (Bharti et al, 2007). Indeed, we demonstrate that recruitment of ASAP1 by PEAK3 is required for the latter to promote a novel invasive phenotype in MCF-10A cells in 3D culture.

Consistent with a previous study (Lopez et al., 2019), we determined that dimerization is required for Crkll binding by PEAK3. However, a striking finding was that this requirement extended more generally to the scaffolding function of PEAK3, with recruitment of Grb2, ASAP1 and PYK2 to PEAK3 also lost upon mutation of the PEAK3 dimerization interface. A detailed understanding of this will require structural studies, but it seems likely that a number of mechanisms underpin this finding. First, the dependency of PEAK3 Y24 phosphorylation on dimerization, since the Y24 SH2 binding site is required for optimal Crkll/Grb2 binding. Here a similar mechanism to that regulating PEAK2 tyrosine phosphorylation may apply, where dimerization activates an associated tyrosine kinase, which in the case of PEAK2 is Csk (Lecointre et al., 2018; Patel et al., 2017; Senda et al., 2016). Based on mutational studies, binding of this kinase to PEAK3 is directly or indirectly mediated via $\mathrm{SH} 3(1)$. Second, dimerization may lead to generation of binding interfaces not present in the individual monomers. Third, the ability of the bridging adaptors to dimerize, which has been demonstrated for Grb2 (Benfield et al, 2007), and is likely to occur with Crkll, based on studies on the closely-related CrkL (Harkiolaki et al, 2006). In this binding mode, significant co-operativity is likely to be observed for binding of the adaptors to the PEAK3 dimer versus the monomer. In addition, it should be noted that particular downstream effectors can also dimerize, including Cbl (Shivanna et al, 2015) and ASAP1 (Chen et al, 2020).

PEAK1 qualitatively regulates tyrosine kinase signal output, mediating a 'switch' in Shc1 signalling from mitogenic/survival signalling at early time points following EGF stimulation to control of cell 
morphology/migration at later times (Zheng et al., 2013). This 'switch' also involves PTPN12, which dephosphorylates the early phase Shc1/Grb2 complex prior to recruitment of PEAK1 to Shc1. In addition, PEAK1 exerts quantitative effects on signalling, promoting aberrant proliferation, migration and invasion when overexpressed (Croucher et al., 2013) and exhibiting upregulation in several cancer types (Agajanian et al., 2015; Croucher et al., 2013; Ding et al., 2018; Huang et al., 2018; Kelber et al., 2012). Importantly, our study reveals interesting similarities between PEAK1 and PEAK3 that extend beyond dimerization and adaptor protein recruitment. Upon EGF treatment, PEAK3 was rapidly dephosphorylated in a PTPN12-dependent manner, highlighting an important role for this PTP in regulating assembly of PEAK family signalling complexes. Since PEAK3 tyrosine phosphorylation regulates Grb2/ASAP1 recruitment, this is consistent with establishing proliferative/survival signals rather than pro-migratory ones during the early phase of EGF receptor signalling (Zheng et al., 2013). In addition, PEAK3 can promote aberrant signalling when overexpressed, leading to failure in the basement membrane integrity of MCF-10A acini and cellular invasion into the surrounding matrix. Given this finding, it will be important to characterize PEAK3 expression in specific cancers and determine whether its downstream pathways represent therapeutic targets.

\section{Materials and Methods}

\section{Plasmids}

Codon-optimized cDNAs encoding N-terminal HA or Flag-tagged WT PEAK3 and HA-tagged PEAK3 mutants were synthesized by Genscript and cloned into the EcoRI restriction sites of the pMIG-GFP Express vector.

\section{Antibodies and reagents}

The following antibodies were used in the study: The PEAK2 antibody was custom-made (Tactacan et al., 2015), as was the PEAK3 antibody (Cambridge, Project Code: CRB240, antigen: SSPEPPTEPPEPDNPTW), PEAK1 (Santa Cruz Biotechnology, cat. sc-100403) ; HA (Cell Signalling, cat. 3724), Flag (Sigma, cat. F1804), p-Tyr (Cell Signalling, cat. 8954S), PYK2 (Cell Signalling, cat. 3480S), Cbl (Santa Cruz Biotechnology, cat. sc-170), EGFR (Cell Signalling, cat. 4267S), Crkll (Santa Cruz Biotechnology, cat. sc-289), Shc (BD Biosciences, cat. 610879), p-Shc (Cell Signalling, cat. 2431S), ASAP1 (Santa Cruz Biotechnology, cat. sc-11539), Ki67 (Cell Signalling, cat. 9027), 14-3-3 (Santa Cruz Biotechnology, cat. sc-1657), PTPN12 (Abcam, cat. ab76942), p-Src Y416 (Cell Signalling, cat. 2101S), $\beta$-actin (Santa Cruz Biotechnology, cat. Sc-69879), Laminin V (Chemicon, cat. MAB19562). AlexaFluor-conjugated (Invitrogen) and HRP-linked (Bio-Rad) secondary antibodies against rabbit and mouse IgG were used in Immunofluorescence staining and Western blotting, respectively. eCF506 was obtained from Professor Margaret Frame (Edinburgh Cancer Research Centre) (Fraser et al., 2016). Dasatinib was purchased from Bristol Myers Squibb (BMS-354825-03). Two synthetic 7 mer peptide variants, either phosphorylated or non-phosphorylated at the key tyrosine residue, were purchased from Mimotopes (pY: Ac-T(pY)SNLGQ-NH2; Y: Ac-TYSNLGQ-NH2; $\mathrm{N}$-terminally acetylated, C-terminally amidated; purity $\geq 95 \%$ ).

Tissue culture and generation of stable cell lines 
MDA-MB-231 cells were obtained from EG\&G Mason Research Institute, Worcester, MA and maintained in RPMI-1640 medium (Gibco, cat. 31800089) supplemented with 10\% FBS (Moregate, cat. MORFBSFAU, Batch: 50301112), $10 \sqsupset \mu \mathrm{g} / \mathrm{mL}$ Actrapid penfill insulin (Clifford Hallam Healthcare), and $20 \sqsupset$ mM HEPES (Life Technologies, cat. 15630080). The MCF-10A cell line stably expressing the murine ecotropic receptor (MCF-10A EcoR) was a kind gift from Drs. Danielle Lynch and Joan Brugge, Harvard Medical School. MCF-10A EcoR cells were maintained as previously described (Brummer et al, 2006). RH30 cells were maintained in RPMI-1640 medium supplemented with 10\% FBS (Moregate). HEK293, PlatE, A172 and SK-N-BE2 cells were maintained in DMEM (Gibco, cat.1200046) supplemented with 10\% FBS (Moregate). PEAK1 and PEAK2 knock-out (KO) MCF-10A cells were generated by CRISPR as previously described (Liu et al., 2016) and double knock-out (DKO) cells were generated by knocking out PEAK1 in PEAK2 KO cells. PEAK3 knock-out (KO) MDA-MB-231 and RH30 cells were generated by CRISPR. The single guide RNAs targeting PEAK3 were designed using GPP sgRNA Designer (Doench et al, 2016) and the sequences were gRNA1: CGCCTCGGAGACCCTTTCTG, gRNA2: GCCCACACGAACTCCTCCGG and gRNA3: GCCCACACGAACTCCTCCGG. All cell lines were authenticated by one or more of short tandem repeat polymorphism, single-nucleotide polymorphism and fingerprint analyses and underwent routine mycoplasma testing by PCR.

\section{Cell lysis and immunoprecipitation}

Cell lysates for immunoblotting and co-immunoprecipitation were prepared using radioimmunoprecipitation (RIPA) buffers and normal lysis buffers, respectively (Brummer et al., 2006). Cell lysates for denatured immunoprecipitation were prepared using $1 \%$ SDS in PBS, heated at $96{ }^{\circ} \mathrm{C}$ for $10 \mathrm{~min}$ and then diluted to $0.1 \%$ SDS with normal lysis buffer. For immunoprecipitation, cell lysates were incubated with anti-HA affinity-agarose beads (Sigma, cat. E6679) at $4{ }^{\circ} \mathrm{C}$ overnight on a shaking platform. Following extensive washing with ice-cold lysis buffer, the immune complexes were eluted with SDS-PAGE loading buffer and subjected to Western blotting analysis.

\section{Western blot}

Protein concentration was measured using BCA assay (Pierce, cat. 23228), and proteins subsequently separated on $8 \%$ bis-acryl-tris gels, and transferred to polyvinylidene difluoride membranes (Millipore, cat. IPVH00010). The membranes were blocked in 5\% skim milk in TBSTween (TBST) (20 mM Tris pH 7.5, $150 \mathrm{mM} \mathrm{NaCl}, 0.05 \%$ Tween 20) for $1 \mathrm{~h}$ at room temperature, and then incubated with the primary antibodies at $4{ }^{\circ} \mathrm{C}$ overnight and the second antibody at room temperature for $1 \mathrm{~h}$. Western blots were visualized by ChemiDoc ${ }^{\mathrm{TM}}$ Touch Gel Imaging System (BioRad, cat. 1708370) and analysed by Image Lab (Version 5.2.1).

\section{Transfections}

Plasmid transfections were performed using Lipofectamine 3000 (Life Technologies, cat. L3000015) according to the manufacturer instructions. Individual siRNAs were obtained from Dharmacon and applied to cells using DharmaFECT1 (Dharmacon, cat. T-2001-03). PEAK3 siRNAs (LQ-027257-020005) sequences were \#18: GGACAACCCCGCUGAUCAA, \#19: GGUCAGCGUCUCCAUGAUA, 
\#20: GGCACAUCCUGGUCGCCAA, and \#21: GCGGGGACGCCCUGUAUUA. PTPN12 siRNAs (LQ008064-00-0005) sequences are \#11: GGAAUUAAGUUCAGAUCUA, \#12: GUAAUGGCCUGCCG AGAAU, \#13: GGACACUCUUACUUGAAUU and \#14: CGGGAGGUAUUCACUAUGA. Negative control siRNA was ON-TARGETplus Non-targeting pool (Dharmacon, D-001810-10-20).

\section{Identification of PEAK3 binding partners by MS-based proteomics}

Control MCF-10A cells or cells expressing HA-tagged PEAK3 were cultured in -EGF medium (DMEM/F-12 (Gibco) supplemented with $0.5 \sqsupset \mu \mathrm{g} / \mathrm{ml}$ hydrocortisone (Sigma), $100 \sqsupset \mathrm{ng} / \mathrm{ml}$ cholera toxin (Sigma), $10 \sqsupset \mu \mathrm{g} / \mathrm{ml}$ bovine insulin (Sigma) and $2 \%$ horse serum overnight and stimulated or not with EGF $(50 \mathrm{ng} / \mathrm{ml})$ for $5 \mathrm{~min}$, and then were lysed in normal lysis buffer (Brummer et al., 2006). Cell lysate $(5 \mathrm{mg}$ ) was incubated with $60 \mu \mathrm{l}$ of anti-HA affinity-agarose beads slurry (Sigma, cat. E6679) for $4 \mathrm{~h}$ at $4{ }^{\circ} \mathrm{C}$. Following washing with lysis buffer and then wash buffer (Tris $20 \mathrm{mM}, \mathrm{pH} 7.4, \mathrm{NaCl} 150$ $\mathrm{mM}$ ), the immunocomplex was digested with $1 \mu \mathrm{g}$ trypsin (Promega, Madison, WI) in $50 \mathrm{mM}$ ammonium bicarbonate solution. Tryptic digests were acidified with $10 \%$ trifluoroacetic acid (TFA) to pH 2-3, desalted with a C18 column (Thermo Fisher Scientific, Waltham, MA) and eluted with $0.1 \%$ TFA $/ 40 \%$ acetonitrile (ACN). Peptides were dried with a SpeedVac and re-suspended in $2 \%$ ACN/0.1\% formic acid (FA). The resulting peptides were analyzed by LC-MS/MS and raw files processed as previously described (Nguyen et al, 2019). Prism (version 8.4.2) was used to compute fold changes and raw $p$ values using unpaired-two tailed $t$ test. Candidate interacting proteins were defined by applying the following criteria: $p$ value $<0.05$ and a fold change of $>1.5$ against the control IP. The mass spectrometry proteomics data have been deposited to the Proteome-Xchange Consortium via the PRIDE (Perez-Riverol et al, 2019) partner repository with the dataset identifier PXD023687.

\section{Wound healing assay}

MDA-MB-231 cells were seeded in 24-well plates at $2.5 \times 10^{5}$ cells/well in duplicate. Wounds were made by scraping with a $200 \mu \mathrm{l}$ plastic pipette tip across the cell monolayer, and then the cells were cultured in $0.4 \%$ FBS RPMI-1640 medium with $1 \mu \mathrm{g} / \mathrm{ml}$ mitomycin C (Sigma, cat. M4287) to prevent cell division. Images were recorded at 0 and $24 \mathrm{~h}$ after scratching. Wound areas were subsequently quantified using Fiji (ImageJ) software (Version: 2.0.0).

\section{Three-dimensional (3D) cultures of MCF-10As}

To establish 3D cultures, cells were plated into a 96-well plate (Falcon) pre-coated with $35 \mu \mathrm{l}$ of Matrigel (Corning, cat. 354230) at a density of $\sim 800$ cells/well in medium containing $2 \%$ Matrigel (Brummer et al., 2006). Cells were allowed to form acini for up to 12 days and fresh medium was replaced every 3 to 4 days. Photographs of acini were taken using a Leica DFC9000 camera with Leica Application Suite $\mathrm{X}$ software. Acinar size was measured using Fiji (ImageJ) software (Version: 2.0.0).

\section{Immunofluorescence staining of acini}


Acini were fixed with $2 \%$ paraformaldehyde in PBS for 20 min and permeabilised with $0.5 \%$ Triton X100 in PBS for $10 \mathrm{~min}$ at $4{ }^{\circ} \mathrm{C}$. Acini were washed three times with PBS/glycine $(130 \mathrm{mM} \mathrm{NaCl}, 7 \mathrm{mM}$ Na2HPO4, $3.5 \mathrm{mM} \mathrm{NaH2PO4,} 100 \mathrm{mM}$ glycine) for $15 \mathrm{~min}$ each. Acini were blocked for $1.5 \mathrm{~h}$ with primary block solution (PBS, $7.7 \mathrm{mM} \mathrm{NaN3}, 10 \%$ bovine serum albumin, $0.2 \%$ Triton $\mathrm{X}-100,0.05 \%$ Tween-20). Primary antibodies were added in primary block solution overnight at $4{ }^{\circ} \mathrm{C}$, then acini were washed three times with IF buffer (PBS, $7.7 \mathrm{mM} \mathrm{NaN3}, 0.1 \%$ bovine serum albumin, $0.2 \%$ Triton $\mathrm{X}-100,0.05 \%$ Tween-20) for 20 min each with gentle rocking. Alexa Fluor $\AA$ secondary antibodies were added in primary block solution for $45 \mathrm{~min}$ at room temperature. Acini were washed three times with IF buffer for 20 min each with rocking. Acini were washed once with PBS, then mounted onto cover slips with gold antifade reagent with DAPI (Invitrogen, cat. P3693). Images were obtained using a Nikon $\mathrm{C} 1$ inverted confocal microscope.

\section{Cell motility assay}

MDA-MB-231 cells were seeded in 12-well plates at $1.5 \times 10^{4}$ cells/well in duplicate and cultured overnight. Then the cells were cultured in 10\% FBS RPMl-1640 medium with $1 \mu \mathrm{g} / \mathrm{ml}$ mitomycin C (Sigma, cat. M4287) to prevent cell division. Images were photographed at 8 random positions for each condition. Images of each position were taken every $20 \mathrm{~min}$ for $24 \mathrm{~h}$ using a Leica DFC9000 with Leica Application Suite $X$ software. Cell motility was measured using Fiji (ImageJ) software (Version: 2.0.0).

\section{Recombinant protein expression and purification}

Synthetic genes encoding full-length (FL) human Grb2 (Uniprot P62993-1; residues 1-217) and Crkll (Uniprot P46108-1; residues 1-330) were each cloned into a modified pCOLD IV vector (Takara) that contains a N-terminal His TEV cleavage tag by Genscript. Protein expression was carried out in Escherichia coli C41(DE3). Inoculated cultures were grown in Super Broth media $(100 \mu \mathrm{g} / \mathrm{mL}$ ampicillin) at $37^{\circ} \mathrm{C}$ to appropriate density (OD600 0.8-1.0), then the cultures were cooled to $16{ }^{\circ} \mathrm{C}$ and protein expression induced with IPTG $(0.5 \mathrm{mM})$ at $16{ }^{\circ} \mathrm{C}$ for $16-20 \mathrm{~h}$. Cell pellets were harvested and stored at $-80^{\circ} \mathrm{C}$ prior to purification.

For purification of both Grb2-FL and Crkll-FL proteins, bacterial cells were resuspended in lysis buffer (20 mM Tris pH 7.5, $500 \mathrm{mM} \mathrm{NaCl}, 10 \% \mathrm{v} / \mathrm{v}$ glycerol, $5 \mathrm{mM}$ DTT, $5 \mathrm{mM}$ imidazole, $0.1 \%$ Thesit), supplemented with Complete EDTA-free Protease Inhibitor Cocktail (Roche) and lysed by sonication. The supernatant was clarified by centrifugation $\left(45,000 \times \mathrm{g}, 4^{\circ} \mathrm{C}, 30 \mathrm{~min}\right)$, filtered and incubated in batch mode with Ni-NTA resin (Roche) $(1 \mathrm{~mL}$ bed volume per $1 \mathrm{~L}$ cell culture, pre-equilibrated in lysis buffer) for $2 \mathrm{~h}$ at $4{ }^{\circ} \mathrm{C}$. The resin was sequentially washed with $2 \times 25 \mathrm{~mL}$ of lysis buffer (pelleted by centrifugation at $1,500 \times \mathrm{xg}, 4^{\circ} \mathrm{C}, 5 \mathrm{~min}$ ), then resuspended in $40 \mathrm{~mL}$ of wash buffer ( $20 \mathrm{mM}$ Tris $\mathrm{pH}$ 7.5, $500 \mathrm{mM} \mathrm{NaCl}, 10 \%(\mathrm{v} / \mathrm{v})$ glycerol, $5 \mathrm{mM}$ DTT, $5 \mathrm{mM}$ imidazole) and transferred to a gravity column. After extensive washes with wash buffer, the protein was eluted in wash buffer supplemented with $250 \mathrm{mM}$ imidazole, then subjected to size exclusion chromatography (SEC) (Superdex-75 16/600, GE Healthcare) in SEC buffer (20 mM Tris pH 7.5, $200 \mathrm{mM} \mathrm{NaCl}, 5 \% \mathrm{v} / \mathrm{v}$ glycerol, $0.5 \mathrm{mM}$ tris (2- carboxyethyl) phosphine (TCEP)). Fractions containing monomeric forms of Grb2-FL or Crkll-FL 
were cleaved overnight using TEV protease then further purified by anion exchange chromatography (MonoQ 5/50 GL) (Cytiva) using Buffer A (20 mM HEPES pH 7.5, 0.5 mM TCEP) and Buffer B (20 $\mathrm{mM}$ HEPES pH 7.5, $1 \mathrm{M} \mathrm{NaCl}, 0.5 \mathrm{mM}$ TCEP). Fractions containing pure monomeric Grb2-FL or Crkll$\mathrm{FL}$ proteins were pooled, concentrated and flash-frozen for storage at $-80^{\circ} \mathrm{C}$.

\section{Isothermal titration calorimetry (ITC) binding experiments}

ITC binding experiments were conducted using a MicroCal iTC200 instrument (Malvern Instruments). Titrations were conducted at $20^{\circ} \mathrm{C}$ in standard mode (protein in cell, in syringe) and consisted of 19 injections of $2 \mu \mathrm{L}$ peptide solution at a rate of $2 \mathrm{sec} / \mu \mathrm{L}$ at $90 \mathrm{sec}$ time intervals, whilst stirring at 1000 rpm. An initial injection of protein $(1 \mu \mathrm{L})$ was made and discarded during data analysis. Proteins (Grb2-FL or Crkll-FL) were first dialysed overnight at $4^{\circ} \mathrm{C}$ into ITC buffer (20 mM HEPES pH 7.4, 150 $\mathrm{mM} \mathrm{NaCl}, 1 \mathrm{mM}$ TCEP). Peptides were diluted into ITC buffer from $10 \mathrm{mM}$ stock solutions prepared in water. Peptides $(400 \mu \mathrm{M}$, in the syringe) were titrated into protein ( $20 \mu \mathrm{M}$ or $10 \mu \mathrm{M}$, in the cell). Data were fitted to a single binding site model to obtain the stoichiometry $(\mathrm{N})$, the dissociation constant $\left(\mathrm{K}_{\mathrm{D}}\right)$ and the enthalpy of binding $(\Delta \mathrm{H})$, using the Microcal Origin software (Version 7.0, OriginLab) provided by the manufacturer. The reported values are the mean \pm S.E.M. from independent measurements (three for the pY peptide into Grb2-FL; four for the pY peptide into Crkll-FL; and two each for the negative control non-phosphorylated peptide into either protein).

\section{Acknowledgements}

This study was supported by funding from the Australian Research Council (DP190103672) and National Health and Medical Research Council (APP1144149). We acknowledge the scientific and technical assistance of Monash Micro Imaging and Monash Biomedical Proteomics Facility, Monash University, Victoria, Australia. We would also like to thank Dr. Jason Cain from Hudson Institute of Medical Research for the A172 cell line.

\section{Author contributions}

Conceptualization: R.J.D. Methodology: J.H., E.V.N., A.S., M.J.R., O.P., I.L., X.M. and R.J.D. Investigation: J.H., E.V.N, A.S., M.J.R., O.P. and X.M. Writing original to final draft: R.D., J.H., and X.M. with input from M.J.R, O.P., and I.L. Review and editing: R.J.D. Funding acquisition: R.J.D and I.L. Project administration and supervision: R.J.D. and X.M.

\section{Conflict of interest}

The authors declare that they have no conflict of interest.

\section{Reference}

Agajanian M, Campeau A, Hoover M, Hou A, Brambilla D, Kim SL, Klemke RL, Kelber JA (2015) PEAK1 Acts as a Molecular Switch to Regulate Context-Dependent TGFbeta Responses in Breast Cancer. PLoS One 10: e0135748

Benfield AP, Whiddon BB, Clements JH, Martin SF (2007) Structural and energetic aspects of Grb2SH2 domain-swapping. Arch Biochem Biophys 462: 47-53 
Bharti S, Inoue H, Bharti K, Hirsch DS, Nie Z, Yoon HY, Artym V, Yamada KM, Mueller SC, Barr VA et al (2007) Src-dependent phosphorylation of ASAP1 regulates podosomes. Molecular and cellular biology 27: 8271-8283

Bristow JM, Reno TA, Jo M, Gonias SL, Klemke RL (2013) Dynamic phosphorylation of tyrosine 665 in pseudopodium-enriched atypical kinase 1 (PEAK1) is essential for the regulation of cell migration and focal adhesion turnover. The Journal of biological chemistry 288: 123-131

Brummer T, Schramek D, Hayes VM, Bennett HL, Caldon CE, Musgrove EA, Daly RJ (2006) Increased proliferation and altered growth factor dependence of human mammary epithelial cells overexpressing the Gab2 docking protein. J Biol Chem 281: 626-637

Chen PW, Billington N, Maron BY, Sload JA, Chinthalapudi K, Heissler SM (2020) The BAR domain of the Arf GTPase-activating protein ASAP1 directly binds actin filaments. The Journal of biological chemistry 295: 11303-11315

Citri A, Yarden Y (2006) EGF-ERBB signalling: towards the systems level. Nat Rev Mol Cell Biol 7: 505-516

Croucher DR, Hochgrafe F, Zhang L, Liu L, Lyons RJ, Rickwood D, Tactacan CM, Browne BC, Ali N, Chan $\mathrm{H}$ et al (2013) Involvement of Lyn and the atypical kinase SgK269/PEAK1 in a basal breast cancer signaling pathway. Cancer research 73: 1969-1980

Debnath J, Muthuswamy SK, Brugge JS (2003) Morphogenesis and oncogenesis of MCF-10A mammary epithelial acini grown in three-dimensional basement membrane cultures. Methods 30 : 256-268

Ding C, Tang W, Fan X, Wang X, Wu H, Xu H, Xu W, Gao W, Wu G (2018) Overexpression of PEAK1 contributes to epithelial-mesenchymal transition and tumor metastasis in lung cancer through modulating ERK1/2 and JAK2 signaling. Cell Death Dis 9: 802

Doench JG, Fusi N, Sullender M, Hegde M, Vaimberg EW, Donovan KF, Smith I, Tothova Z, Wilen C, Orchard $\mathrm{R}$ et al (2016) Optimized sgRNA design to maximize activity and minimize off-target effects of CRISPR-Cas9. Nat Biotechno/34: 184-191

Fraser C, Dawson JC, Dowling R, Houston DR, Weiss JT, Munro AF, Muir M, Harrington L, Webster SP, Frame MC et al (2016) Rapid Discovery and Structure-Activity Relationships of Pyrazolopyrimidines That Potently Suppress Breast Cancer Cell Growth via SRC Kinase Inhibition with Exceptional Selectivity over ABL Kinase. J Med Chem 59: 4697-4710

$\mathrm{Ha}$ BH, Boggon TJ (2018) The crystal structure of pseudokinase PEAK1 (Sugen kinase 269) reveals an unusual catalytic cleft and a novel mode of kinase fold dimerization. $J$ Biol Chem 293: $1642-$ 1650

Harkiolaki M, Gilbert RJ, Jones EY, Feller SM (2006) The C-terminal SH3 domain of CRKL as a dynamic dimerization module transiently exposing a nuclear export signal. Structure 14: 17411753

Huang L, Wen C, Yang X, Lou Q, Wang X, Che J, Chen J, Yang Z, Wu X, Huang M et al (2018) PEAK1, acting as a tumor promoter in colorectal cancer, is regulated by the EGFR/KRas signaling axis and miR-181d. Cell Death Dis 9: 271 
Kelber JA, Reno T, Kaushal S, Metildi C, Wright T, Stoletov K, Weems JM, Park FD, Mose E, Wang Y et al (2012) KRas induces a Src/PEAK1/ErbB2 kinase amplification loop that drives metastatic growth and therapy resistance in pancreatic cancer. Cancer research 72: 2554-2564

Kong R, Feng J, Ma Y, Zhou B, Li S, Zhang W, Jiang J, Zhang J, Qiao Z, Zhang T et al (2016) Silencing NACK by siRNA inhibits tumorigenesis in non-small cell lung cancer via targeting Notch1 signaling pathway. Oncol Rep 35: 2306-2314

Lecointre C, Simon V, Kerneur C, Allemand F, Fournet A, Montarras I, Pons JL, Gelin M, Brignatz C, Urbach $S$ et al (2018) Dimerization of the Pragmin Pseudo-Kinase Regulates Protein Tyrosine Phosphorylation. Structure 26: 545-554 e544

Leroy C, Fialin C, Sirvent A, Simon V, Urbach S, Poncet J, Robert B, Jouin P, Roche S (2009) Quantitative phosphoproteomics reveals a cluster of tyrosine kinases that mediates SRC invasive activity in advanced colon carcinoma cells. Cancer Res 69: 2279-2286

Liu L, Phua YW, Lee RS, Ma X, Jenkins Y, Novy K, Humphrey ES, Chan H, Shearer R, Ong PC et al (2016) Homo- and Heterotypic Association Regulates Signaling by the SgK269/PEAK1 and SgK223 Pseudokinases. The Journal of biological chemistry 291: 21571-21583

Lopez ML, Lo M, Kung JE, Dudkiewicz M, Jang GM, Von Dollen J, Johnson JR, Krogan NJ, Pawlowski K, Jura N (2019) PEAK3/C19orf35 pseudokinase, a new NFK3 kinase family member, inhibits Crkll through dimerization. Proc Natl Acad Sci U S A 116: 15495-15504

Lowenstein EJ, Daly RJ, Batzer AG, Li W, Margolis B, Lammers R, Ullrich A, Skolnik EY, Bar-Sagi D, Schlessinger J (1992) The SH2 and SH3 domain-containing protein GRB2 links receptor tyrosine kinases to ras signaling. Cell 70: 431-442

Manning G, Whyte DB, Martinez R, Hunter T, Sudarsanam S (2002) The protein kinase complement of the human genome. Science 298: 1912-1934

Miller ML, Jensen LJ, Diella F, Jorgensen C, Tinti M, Li L, Hsiung M, Parker SA, Bordeaux J, Sicheritz-Ponten T et al (2008) Linear motif atlas for phosphorylation-dependent signaling. Science signaling 1: ra2

Nguyen EV, Pereira BA, Lawrence MG, Ma X, Rebello RJ, Chan H, Niranjan B, Wu Y, Ellem S, Guan $X$ et al (2019) Proteomic Profiling of Human Prostate Cancer-associated Fibroblasts (CAF) Reveals LOXL2-dependent Regulation of the Tumor Microenvironment. Molecular \& cellular proteomics : MCP 18: 1410-1427

Patel O, Griffin MDW, Panjikar S, Dai W, Ma X, Chan H, Zheng C, Kropp A, Murphy JM, Daly RJ et al (2017) Structure of SgK223 pseudokinase reveals novel mechanisms of homotypic and heterotypic association. Nat Commun 8: 1157

Patel O, Roy MJ, Murphy JM, Lucet IS (2020) The PEAK family of pseudokinases, their role in cell signalling and cancer. The FEBS journal 287: 4183-4197

Perez-Riverol Y, Csordas A, Bai J, Bernal-Llinares M, Hewapathirana S, Kundu DJ, Inuganti A, Griss $J$, Mayer G, Eisenacher $M$ et al (2019) The PRIDE database and related tools and resources in 2019: improving support for quantification data. Nucleic Acids Res 47: D442-D450

Reiterer V, Eyers PA, Farhan H (2014) Day of the dead: pseudokinases and pseudophosphatases in physiology and disease. Trends Cell Biol 24: 489-505 
Safari F, Murata-Kamiya N, Saito Y, Hatakeyama M (2011) Mammalian Pragmin regulates Src family kinases via the Glu-Pro-Ile-Tyr-Ala (EPIYA) motif that is exploited by bacterial effectors. Proc Natl Acad Sci U S A 108: 14938-14943

Senda Y, Murata-Kamiya N, Hatakeyama M (2016) C-terminal Src kinase-mediated EPIYA phosphorylation of Pragmin creates a feed-forward C-terminal Src kinase activation loop that promotes cell motility. Cancer Sci 107: 972-980

Shivanna S, Harrold I, Shashar M, Meyer R, Kiang C, Francis J, Zhao Q, Feng H, Edelman ER, Rahimi $\mathrm{N}$ et al (2015) The c-Cbl ubiquitin ligase regulates nuclear beta-catenin and angiogenesis by its tyrosine phosphorylation mediated through the Wnt signaling pathway. J Biol Chem 290: 12537-12546

Tactacan CM, Phua YW, Liu L, Zhang L, Humphrey ES, Cowley M, Pinese M, Biankin AV, Daly RJ (2015) The pseudokinase SgK223 promotes invasion of pancreatic ductal epithelial cells through JAK1/Stat3 signaling. Mol Cancer 14: 139

Tanna CE, Goss LB, Ludwig CG, Chen PW (2019) Arf GAPs as Regulators of the Actin CytoskeletonAn Update. Int $J$ Mol Sci 20

Wang Y, Kelber JA, Tran Cao HS, Cantin GT, Lin R, Wang W, Kaushal S, Bristow JM, Edgington TS, Hoffman RM et al (2010) Pseudopodium-enriched atypical kinase 1 regulates the cytoskeleton and cancer progression [corrected]. Proc Natl Acad Sci U S A 107: 10920-10925

Zheng Y, Zhang C, Croucher DR, Soliman MA, St-Denis N, Pasculescu A, Taylor L, Tate SA, Hardy WR, Colwill $\mathrm{K}$ et al (2013) Temporal regulation of EGF signalling networks by the scaffold protein Shc1. Nature 499: 166-171

\section{Figure legends}

Figure 1. PEAK3 undergoes heterotypic association with PEAK1 and PEAK2 via the dimerization interface.

A Schematic representation of PEAK1, PEAK2 and PEAK3 domain structure.

B Schematic representation of key amino acid residues in predicted dimerization interface of PEAK3.

C PEAK3 heterotypic association. HA-tagged WT PEAK3 or dimerization-interface mutants were overexpressed in parental, PEAK1 KO or PEAK2 KO MCF-10A cells by retroviral infection and empty vector was used as a control. Lysates were subjected to immunoprecipitation with an anti-HA antibody and Western blotted as indicated. Data are representative of three independent experiments. TCL: total cell lysate.

\section{Figure 2. Characterization of PEAK3 binding partners.}

A Interactomes of PEAK3 under -/+ EGF stimulation represented as volcano plots of the MS data. MCF-10A cells stably overexpressing HA-tagged PEAK3 or empty vector were cultured in -EGF medium as described in Methods overnight and stimulated with EGF $(50 \mathrm{ng} / \mathrm{ml})$ for $5 \mathrm{~min}$. Anti-HA IPs were prepared and subjected to on-beads tryptic digestion. Resulting peptides were subjected to LC$\mathrm{MS} / \mathrm{MS}$ analysis. Dashed lines indicate the $\mathrm{p}<0.05$ and 1.5 -fold change $(\log 2(\mathrm{FC})=0.58$, up for 
PEAK3 versus Vector, up and down for EGF versus No EGF) thresholds. Most pronounced responding proteins are indicated in red dots and labelled.

B, C Validation of MS results by Western blot. Anti-HA IPs were prepared using DKO MCF-10A cells stably overexpressing HA-tagged PEAK3 or empty vector treated with EGF for different time points, and Western blotted as indicated. Data are representative of at least three independent experiments. Predicted tyrosine phosphorylated proteins corresponding to the bands are indicated in (B).

\section{Figure 3. Regulation of PEAK3 tyrosine phosphorylation.}

A Effect of PTPN12 knockdown on PEAK3 tyrosine phosphorylation. PTPN12 siRNA pool (20 nM) was transfected into DKO MCF-10A cells stably overexpressing HA-tagged PEAK3, after $48 \mathrm{~h}$ of transfection the cells were starved in -EGF medium overnight and treated with EGF ( $50 \mathrm{ng} / \mathrm{ml})$ for 5 min. Non-targeting siRNA pool (OTP) was used as control.

B Effect of Src inhibition on PEAK3 tyrosine phosphorylation. DKO MCF-10A cells stably overexpressing HA-tagged PEAK3 were starved in -EGF medium overnight and treated with SrC inhibitors eCF506 (250 nM) or Dasatinib (100 nM) for $1 \mathrm{~h}$.

C Schematic representation of mutated $\mathrm{SH} 2$ and $\mathrm{SH} 3$ domain binding motifs in PEAK3.

D Effect of EGF treatment and mutation of $\mathrm{SH} 2$ and $\mathrm{SH} 3$ binding motifs on PEAK3 tyrosine phosphorylation. DKO MCF-10A cells stably overexpressing HA-tagged WT PEAK3 or specific mutants were starved in -EGF medium overnight and treated with EGF $(50 \mathrm{ng} / \mathrm{ml})$ for $5 \mathrm{~min}$.

A, B and D Anti-HA IPs were prepared from denatured cell lysates and Western blotted as indicated. Data are representative of at least three independent experiments.

Figure 4. PEAK3 dimerization and interaction with binding partners.

A Effect of dimerization on PEAK3 association with specific binding partners. HEK293 cells were cotransfected with plasmids encoding Flag-tagged WT PEAK3 in combination with plasmids encoding HA-tagged WT PEAK3 or dimerization-deficient mutants.

B Effect of mutated SH2 and SH3 domain binding motifs on PEAK3 dimerization. HEK293 cells were co-transfected with plasmids encoding Flag-tagged WT PEAK3 in combination with plasmids encoding HA-tagged WT or specific mutant versions of PEAK3.

C Role of PEAK3 SH2 and SH3 domain binding motifs in PEAK3 association with specific binding partners. HA-tagged WT PEAK3 or specific mutants were overexpressed in DKO MCF-10A cells by retroviral infection.

A, B and C Empty vector was used as a control. Lysates were subjected to immunoprecipitation with an anti-HA antibody and Western blotted as indicated. Data are representative of three independent experiments.

Figure 5. PEAK3 binding with Grb2 and Crkll. 
A ITC data for binding of PEAK3 phosphorylated peptide (T(pY)SNLGQ) and non-phosphorylated peptide (TYSNLGQ) with full-length Grb2 (Grb2-FL). The equilibrium dissociation constant $\left(\mathrm{K}_{\mathrm{D}}\right)$ for the interaction of Grb2 with the PEAK3 peptide-pY was $2.6 \pm 0.2 \mu \mathrm{M}$ from three replicate experiments.

B ITC data for the binding of PEAK3 phosphorylated peptide (T(pY)SNLGQ) and non-phosphorylated peptide (TYSNLGQ) with full-length Crkll (Crkll-FL). The $K_{D}$ of the interaction of Crkll with the PEAK3 peptide-pY was $7.8 \pm 0.4 \mu \mathrm{M}$ from four replicate experiments.

C Data of $A$ and $B$ were fitted to a single binding site model to obtain the stoichiometry $(N)$, the dissociation constant $\left(K_{D}\right)$ and the enthalpy of binding $(\Delta \mathrm{H}) . \Delta \mathrm{G}$ is Gibbs free energy change on binding, $\Delta S$ is the entropy change on binding. The reported values are the mean \pm s.e.m. from independent measurements as indicated.

D Effect of Grb2 knockdown on PEAK3 association with specific binding partners and tyrosine phosphorylation. Grb2 siRNA pool (20 nM) was transfected into DKO MCF-10A cells stably overexpressing HA-tagged PEAK3. Non-targeting siRNA pool (OTP) was used as control. Anti-HA IPs were prepared and Western blotted as indicated. In the right-hand panel, IPs were prepared from denatured lysates. Data are representative of at least three independent experiments.

\section{Figure 6. Effect of PEAK3 on elongation and motility in MDA-MB-231 cells.}

A Expression levels of HA/PEAK3 and mutants in MDA-MB-231 cells. Lysates prepared from cells stably expressing HA-tagged WT PEAK3 and mutants were Western blotted as indicated.

$B, C$ Effect of WT PEAK3 and different mutants on elongation in MDA-MB-231 cells. MDA-MB-231 cells in (A) were used for morphology analysis and quantification of the percentage of elongated cells. Representative images of WT PEAK3-expressing cells are shown in (B). Scale bar, $50 \mu \mathrm{m}$. Data in (C) represent the mean \pm s.e.m. of three independent experiments. At least 1000 cells were analyzed in each experiment.

D, E Effect of WT PEAK3 on random cell motility in MDA-MB-231 cells. HA-tagged WT PEAK3 was stably overexpressed in MDA-MB-231 cells and random cell motility was determined by live cell tracking. Representative images are shown in (D). The histogram in $(E)$ indicates the average maximum displacement distance from the origin. Scale bar, $200 \mu \mathrm{m}$. Data represent the mean \pm s.e.m. of three independent experiments. $\sim 60$ cells were analyzed in each experiment.

F, G Knockdown of endogenous PEAK3 inhibits MDA-MB-231 cell migration. PEAK3 individual siRNAs 18-21 (20 nM) were transfected into MDA-MB-231 cells. Cell lysates were Western blotted (F) and a wound healing assay was performed $(G)$. OTP was a non-targeting control. The histogram in (G) provides the quantitative analysis of the relative wound area for 4 different PEAK3 siRNAs. *, $\mathrm{p}<0.05,{ }^{* *}, \mathrm{p}<0.01,{ }^{* * *}, \mathrm{p}<0.001,{ }^{* * * *}, \mathrm{p}<0.0001$, by Student's t-test. The representative images in $(\mathrm{G})$ were taken immediately after scratches had been made and after $24 \mathrm{~h}$ incubation. The black lines indicate the wound area. Scale bar, $200 \mu \mathrm{m}$.

Figure 7. Effect of PEAK3 on MCF-10A acini growth. 
A Expression levels of HA/PEAK3 and mutants in DKO MCF-10A cells. Lysates prepared from stably expressing cells were Western blotted.

B, C Effect of WT PEAK3 and specific mutants on MCF-10A acini growth. DKO MCF-10A cells in (A) were used for $3 \mathrm{D}$ culture. Micrographs, acini grown on Matrigel for 5 days. Scale bar, $100 \mu \mathrm{m}$. Histogram, quantification of the relative average size of acini expressing PEAK3 WT and the different mutants.

D, E Fluorescence microscopy of acini grown on Matrigel for 12 days and stained with Ki67 antibody (red) and nuclei visualized with DAPI (blue), subsequently analysed by confocal microscopy. Histogram, quantification of Ki67 positive acini. Data represent the mean \pm s.e.m. of 3 independent experiments. $\geq 50$ acini were analyzed in each experiment. ${ }^{*}, p<0.05,{ }^{* *}, p<0.01,{ }^{* * *}, p<0.0001$, by Student's t-test.

\section{Figure 8. Effect of PEAK3 on MCF-10A acini invasion.}

A Effect of WT PEAK3 on MCF-10A acini invasion. Micrographs of vector control and PEAK3 overexpressing DKO MCF-10A acini grown on Matrigel for 12 days. Red arrowheads indicate invasive cells. Scale bar, $100 \mu \mathrm{m}$.

B Fluorescence microscopy indicate acini with invasive phenotype, stained with antibody against laminin V (red) and nuclei visualized with DAPI (blue), and subsequently analysed by confocal microscopy. Red arrowheads indicate invasive cells. Scale bar, $100 \mu \mathrm{m}$.

C Effect of WT PEAK3 and specific mutants on MCF-10A acini invasion. DKO MCF-10A cells overexpressing WT PEAK3 and specific mutants were maintained in 3D culture for 12 days. The histogram indicates the quantification of the number of acini with invasive phenotype per 100 acini. Data represent the mean \pm s.e.m. of three independent experiments. ${ }^{*}, p<0.05,{ }^{* * *}, p<0.001,{ }^{* * * *}$, $\mathrm{p}<0.0001$, by Student's t-test.

\section{Table legend}

\section{Table EV1. Lists of PEAK3 binding partners.}

PEAK3 binding partners were identified by MS from MCF-10A_Control and MCF-10A_PEAK3 cells as described in Methods. Left list: The 109 proteins exhibiting significantly increased abundance in PEAK3 IPs versus control IPs. Right list: The 29 PEAK3 binding partners regulated by EGF treatment, with 7 up-regulated and 22 down-regulated.

\section{Expanded View Figure legends}

Figure EV1. Characterization of PEAK1 KO, PEAK2 KO and DKO MCF-10A cells generated by CRISPR/Cas9 and data quantification of PEAK3 heterotypic association.

A Western blotting was undertaken as indicated.

B Quantification of Western blots in Figure 1C (normalized to HA) and data expressed relative to WT PEAK3 which was arbitrarily set at 1 . Error bars represent s.e.m., ${ }^{* *}, p<0.01,{ }^{* * *}, p<0.0001, \#$, $p>0.05$ by Student's t-test. ND, not detected. 
Figure EV2. The dynamics of the PEAK3 interactome after EGF treatment. $X$ axis indicates EGF treatment time points $(50 \mathrm{ng} / \mathrm{ml})$. Levels of associated binding partners are normalized for the amount of HA-tagged PEAK3 in the IP and expressed as a percentage of their highest level over the time course.

Figure EV3. A model of the full length PEAK3. The pseudokinase domain is adopted from the crystal structure of PEAK2 (PDB 5VE6) dimer. SH2 and SH3 motifs within the PEST linker region and the pseudokinase domain are as shown. The position of a-turn (red) in PEAK1 and PEAK2 corresponds to the SH3(2) motif in PEAK3, PGAPWR. The interaction triad between Phe974 of aN1, Phe1045 of loop connecting $\beta 3-\beta 4$ (black) and Trp1382 of $\alpha \mathrm{L}$ is shown in a green circle. In PEAK3, Phe974 is replaced by a leucine and is likely to maintain this interaction triad. SH3(2) mutation in PEAK3 may impact the orientation of the $\mathrm{N}$-lobe with respect to the C-lobe, affecting this interaction triad and hence dimerisation. Grb2 and Crkll binding through their SH2 and SH3 domains is shown on the SH2 and SH3(1) motifs. Unresolved loops are shown as dashed lines.

\section{Figure EV4. Data quantification.}

A Quantification of Western blots in Figure 4B (normalized to HA).

B Quantification of Western blots in Figure 4C (normalized to HA).

A and B Data expressed relative to WT PEAK3 which was arbitrarily set at 1 . Error bars represent s.e.m., ${ }^{\star}, p<0.05,{ }^{\star *}, p<0.01,{ }^{\star * * *}, p<0.0001, \#, p>0.05$ by Student's t-test. ND, not detected.

C. Proposed model of ASAP1 association. Grb2 bridges PEAK3 to ASAP1 by binding to the SH2 binding motif on PEAK3 and SH3 binding motif(s) on ASAP1. Other potential mechanisms contributing to ASAP1 association with PEAK3 (dotted lines) are bridging by Crkll, which binds to the $\mathrm{SH} 3(1)$ motif and then associates with ASAP1 via SH2 or SH3 domain-mediated interactions, and direct binding via the ASAP1 SH3 domain. Note that integrity of $\mathrm{SH} 3(1)$ is also required for efficient Y24 phosphorylation (red arrow).

\section{Figure EV5. Detection of endogenous PEAK3.}

A Validation of PEAK3 custom antibody. HEK293 cells were transfected with non-tagged PEAK3.

B PEAK3 was knocked out in MDA-MB-231 and RH30 cells by CRISPR/Cas9.

C PEAK3 was knocked down in A172 cells by PEAK3 siRNA pool (20 nM).

Cell lysates from A-C were Western blotted as indicated. 
A

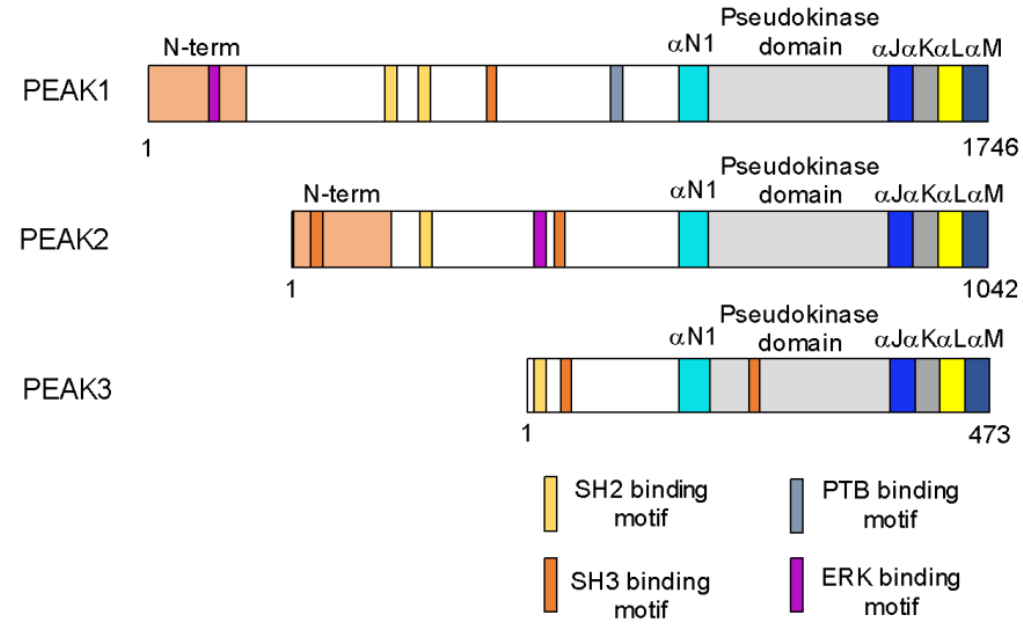

B

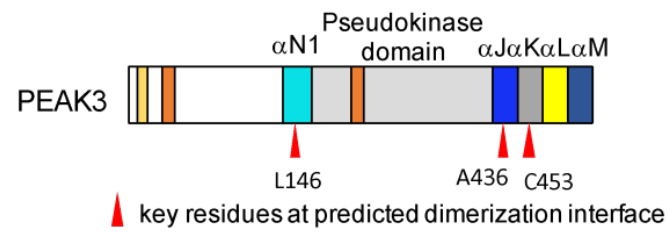

C
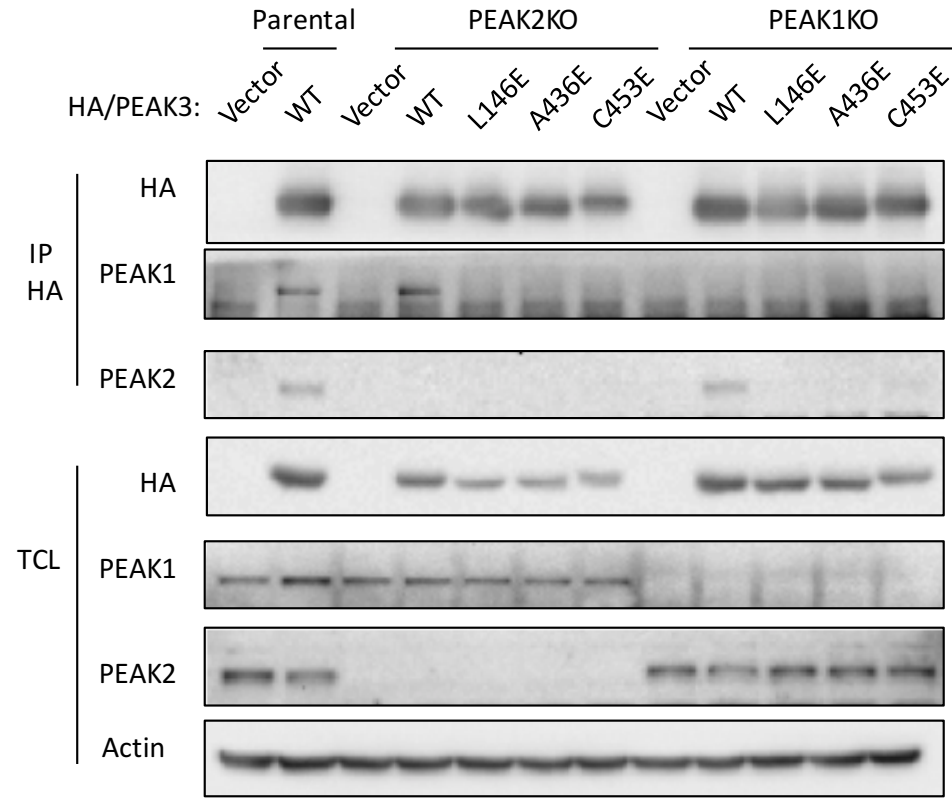

Figure 1 


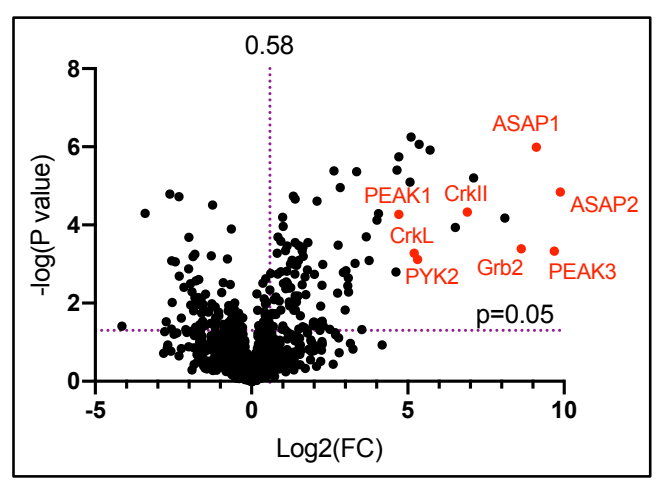

PEAK3 / Vector

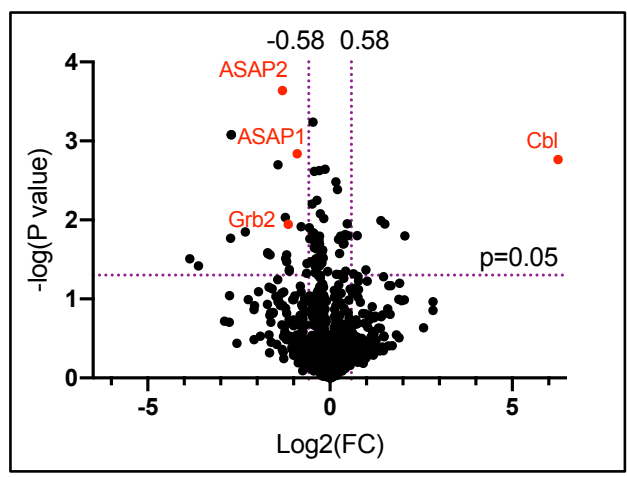

EGF / No EGF

B

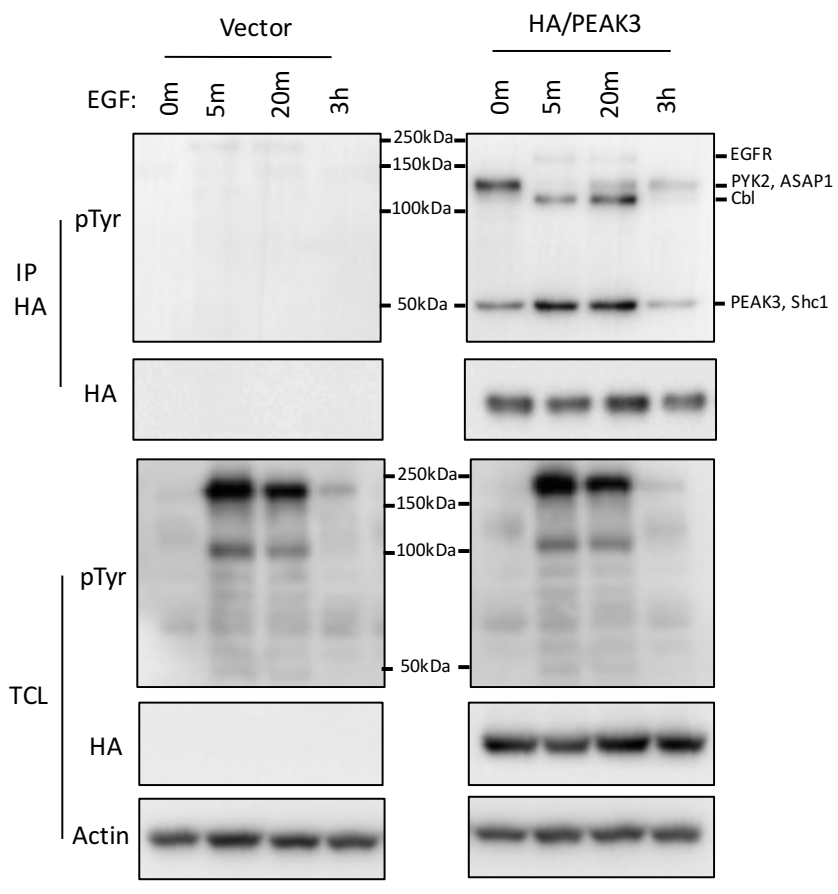

C

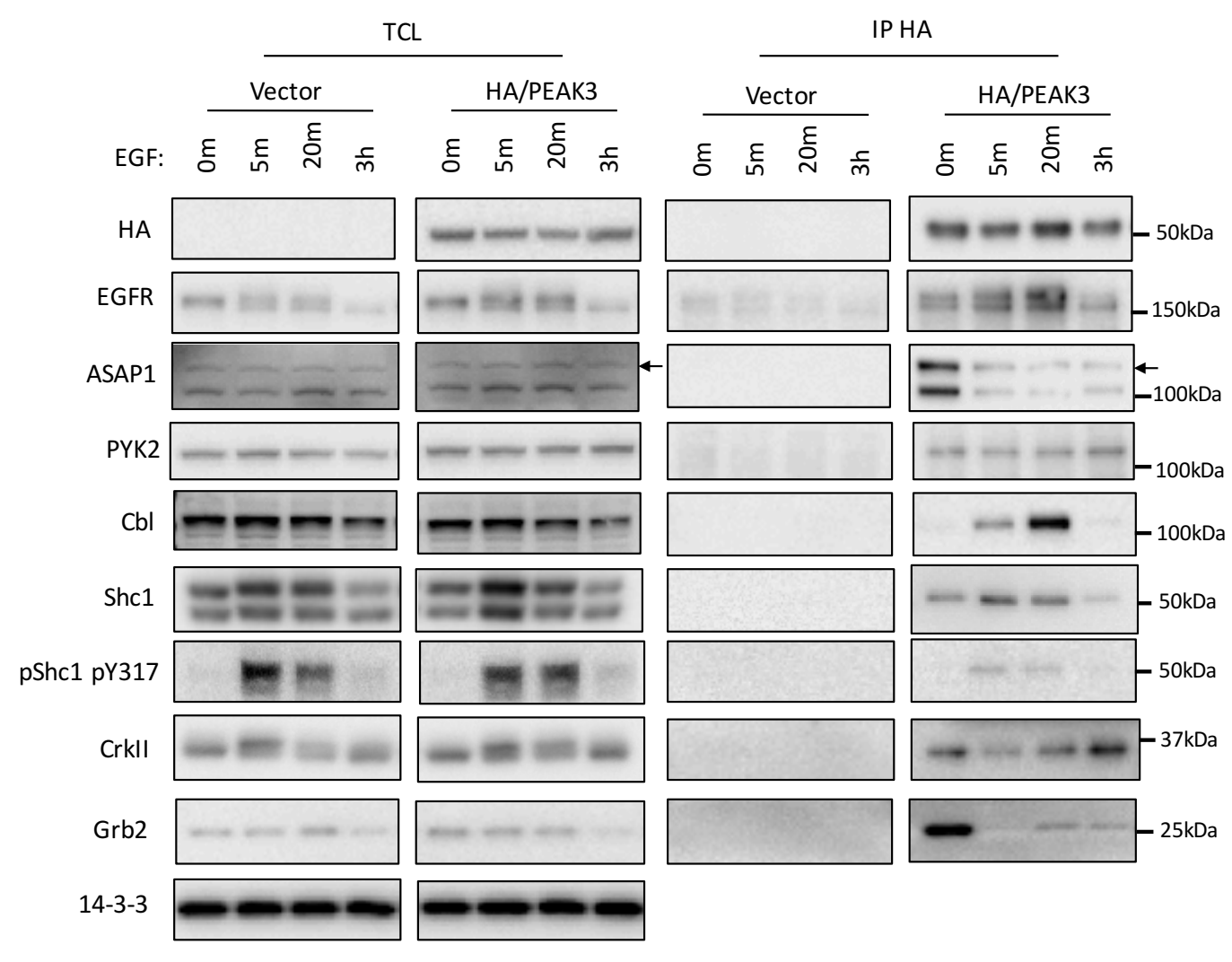


A

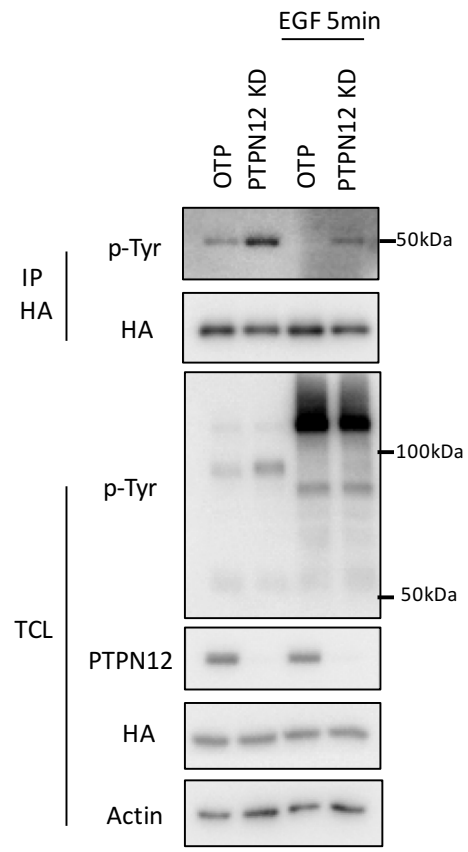

B

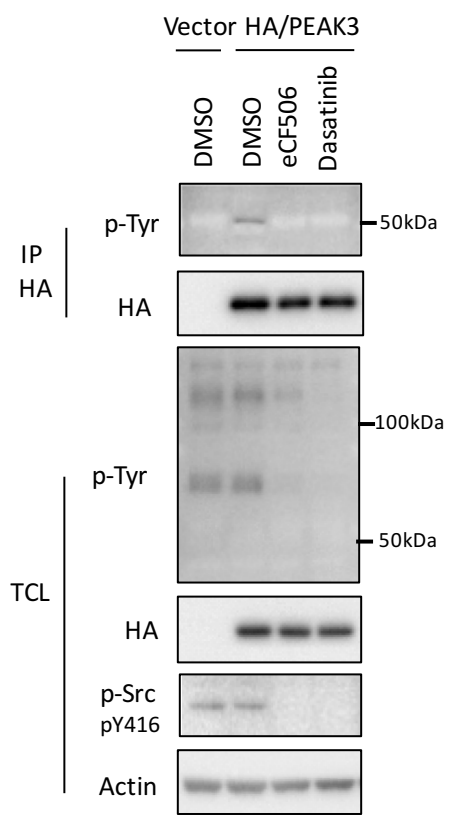

C

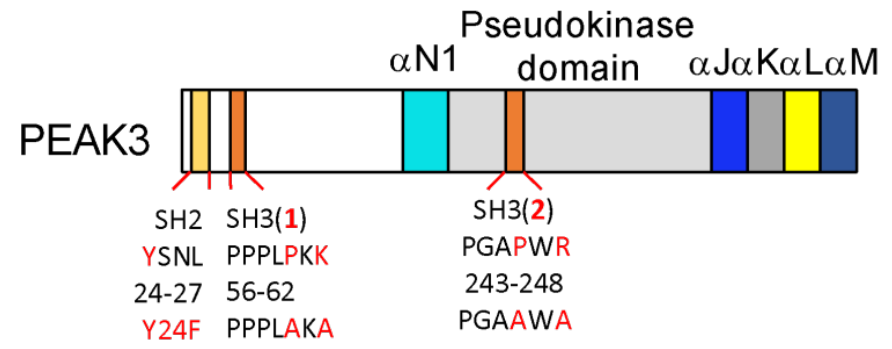

D

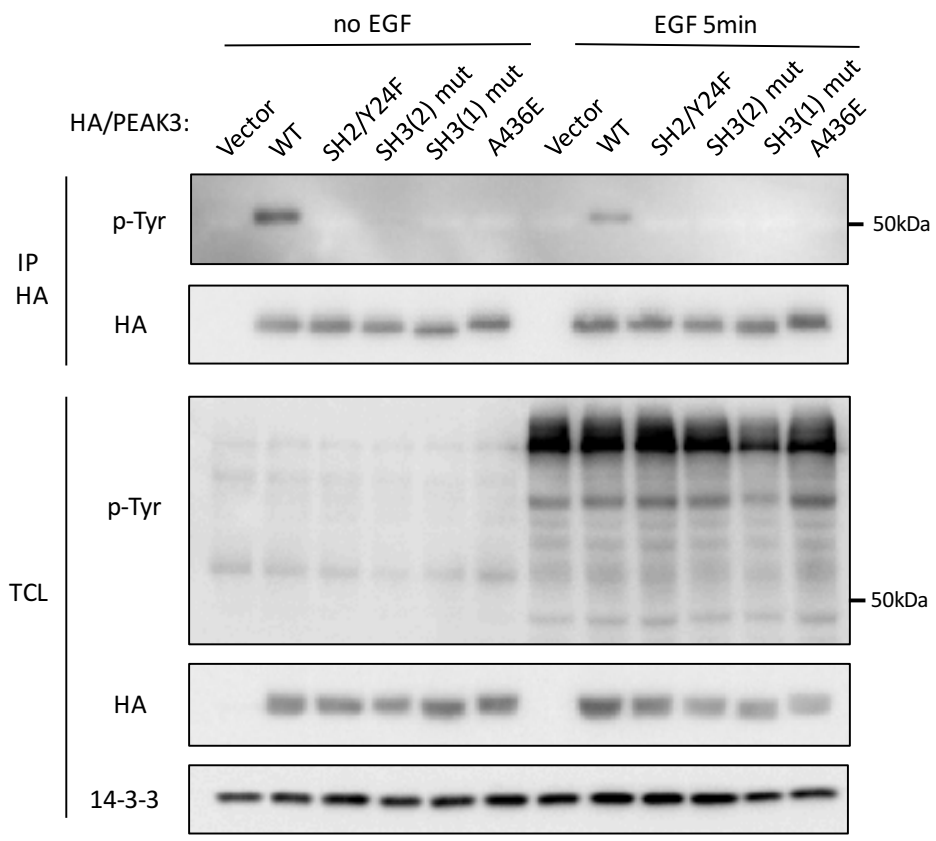

Figure 3 


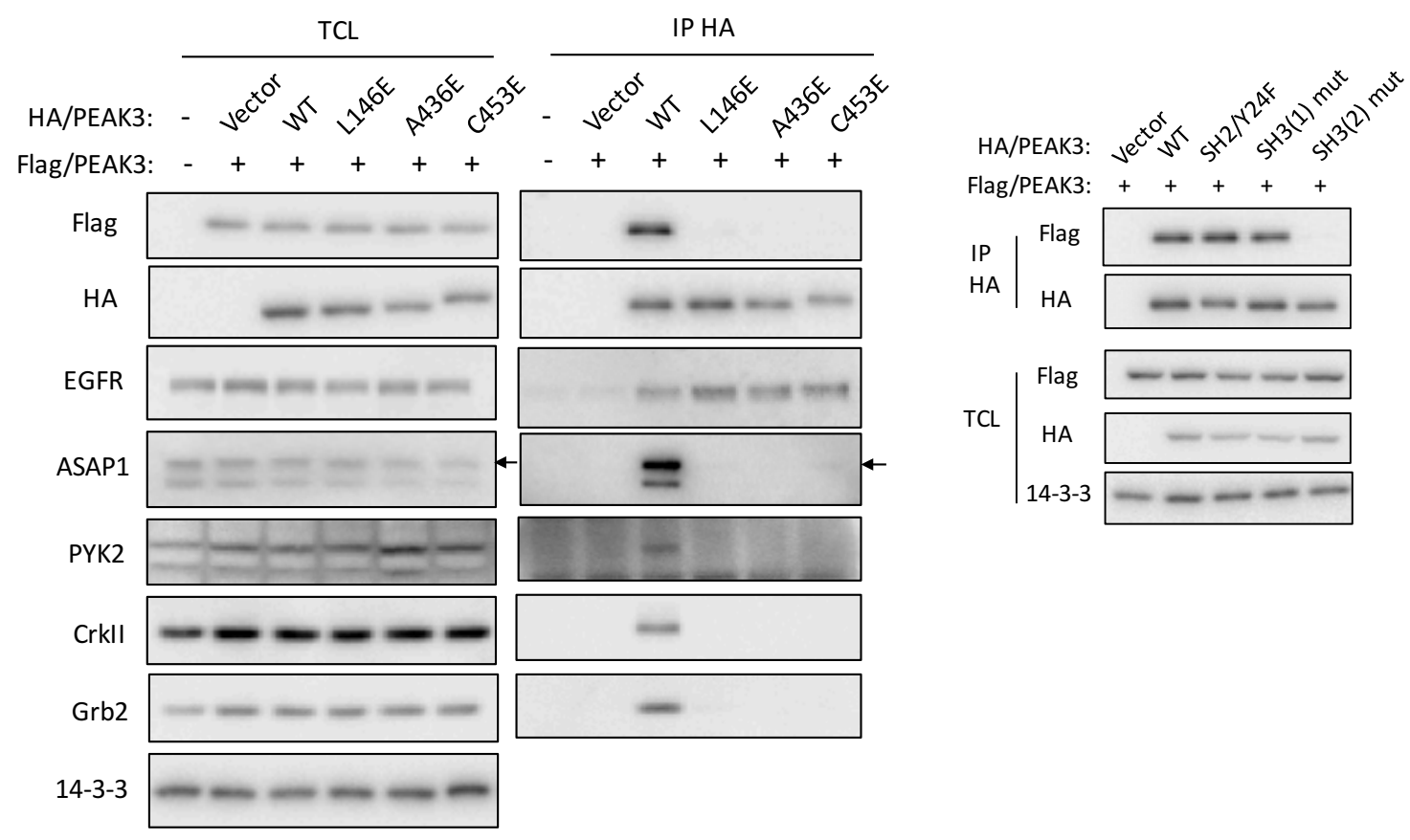

C

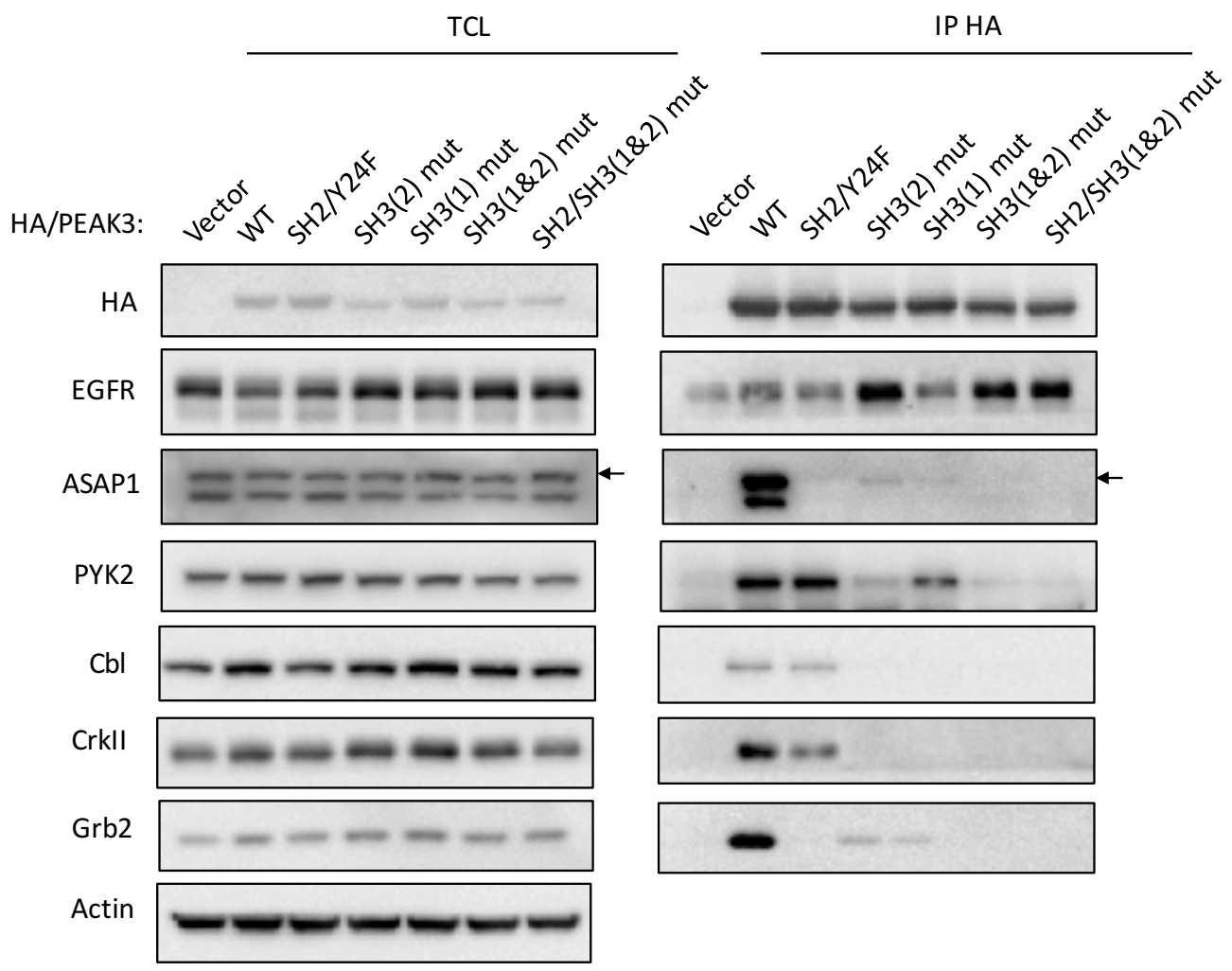

Figure 4 
A

Grb2-FL

(SH2) peptide-pY
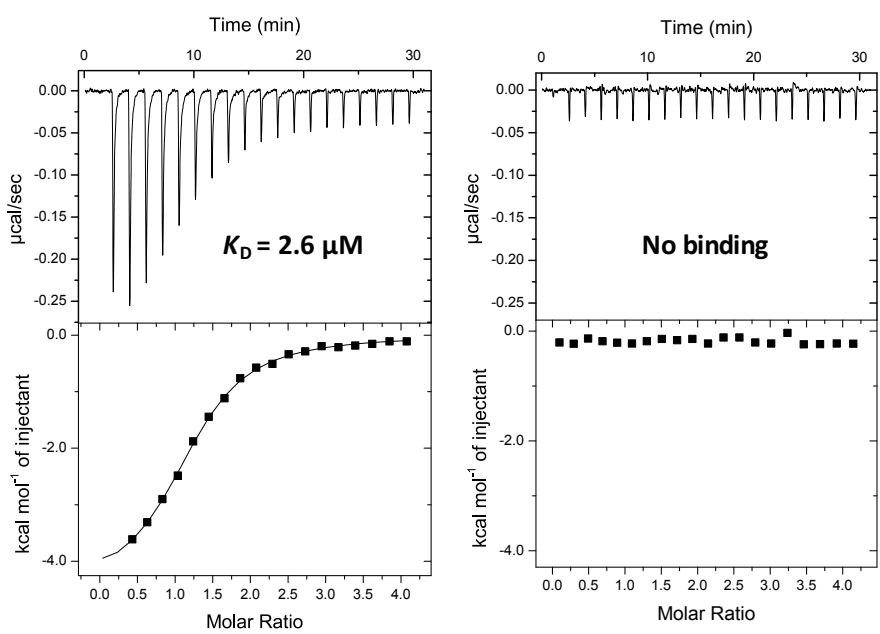

B

CrkII-FL

(SH2) peptide-pY

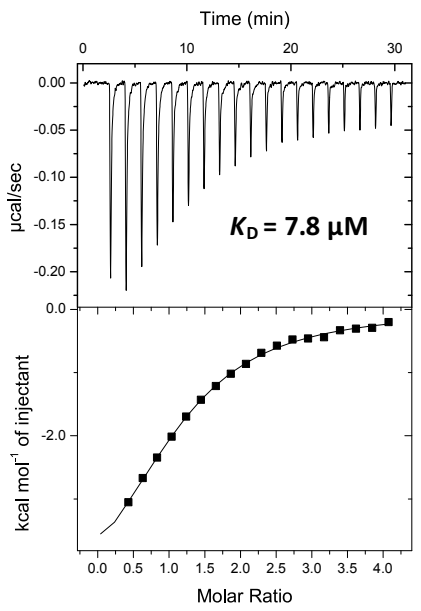

C

\begin{tabular}{|c|c|c|c|c|c|c|c|c|c|c|c|c|}
\hline Protein & Peptide & $\mathrm{N}$ (repeats) & $\mathrm{N}$ (sites) & \pm s.e.m. & $K_{\mathrm{D}}(\mu \mathrm{M})$ & \pm s.e.m. & $\Delta \mathrm{H}$ (kcal $/ \mathrm{mol})$ & \pm s.e.m. & $\Delta \mathbf{G}$ (kcal $/ \mathrm{mol})$ & \pm s.e.m. & $\Delta \mathrm{S}$ (cal/mol/deg) & \pm s.e.m. \\
\hline \multirow{2}{*}{ Grb2-FL } & (SH2) peptide-pY & 3 & 1.1 & 0.1 & 2.6 & 0.2 & -4.7 & 0.5 & -7.5 & 0.1 & 9.6 & 1.8 \\
\hline & (SH2) peptide & 2 & & & No binding & & & & & & & \\
\hline \multirow{2}{*}{ Crkll-FL } & (SH2) peptide-pY & 4 & 1.0 & 0.1 & 7.8 & 0.4 & -5.0 & 0.2 & -6.9 & 0.1 & 6.4 & 0.8 \\
\hline & (SH2) peptide & 2 & & & No binding & & & & & & & \\
\hline
\end{tabular}

D
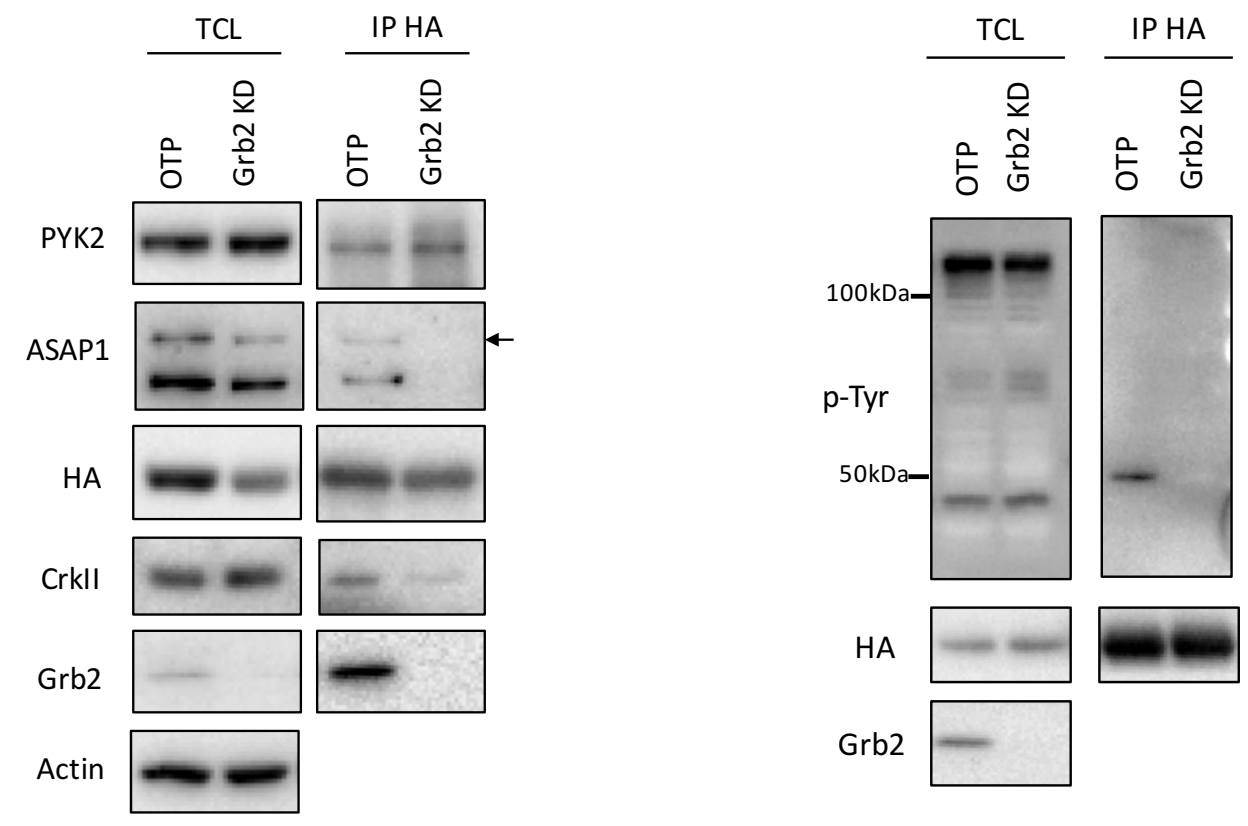

Figure 5 
A

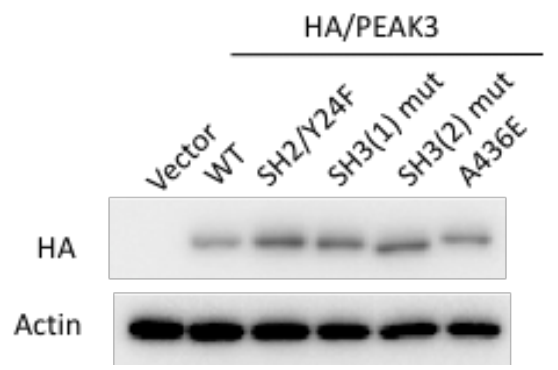

C

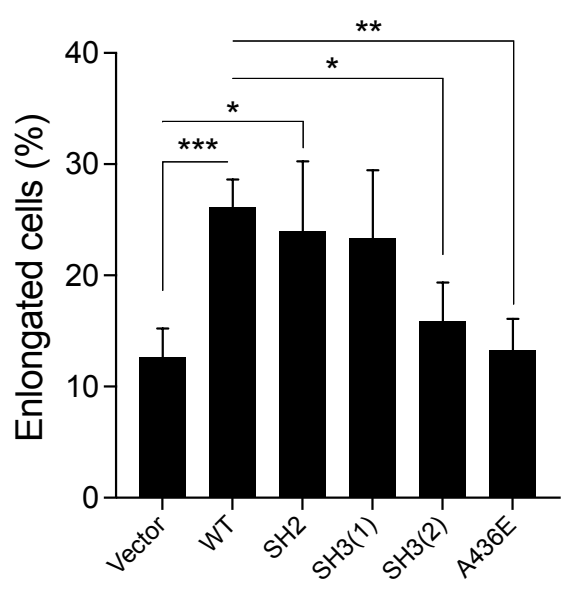

F

G

D
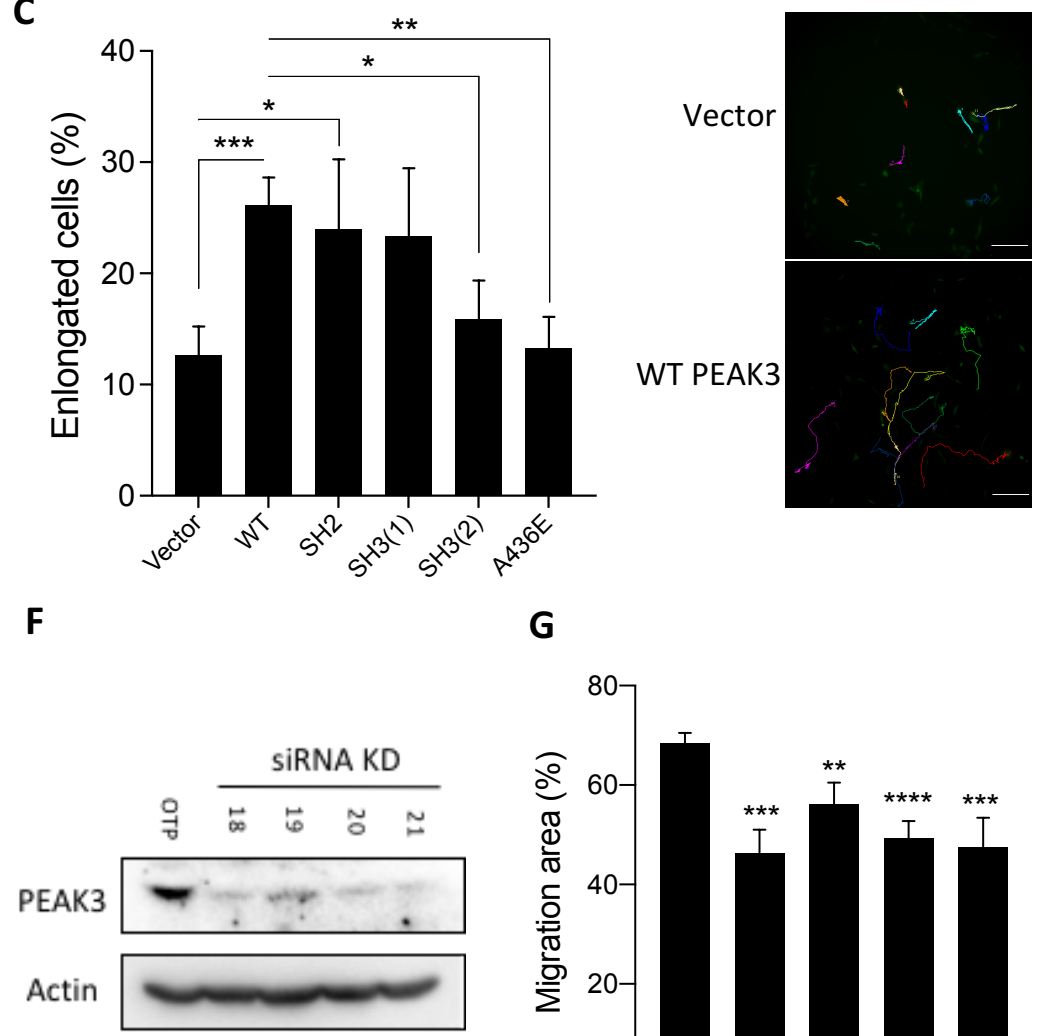

B
Vector

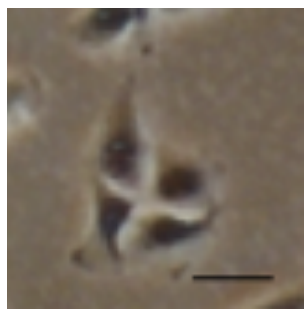

E

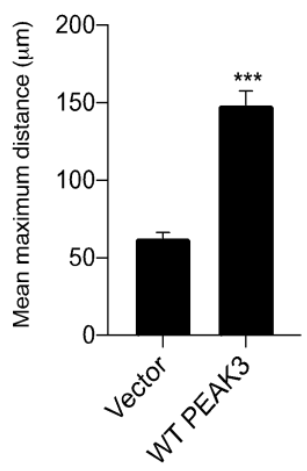

Oh

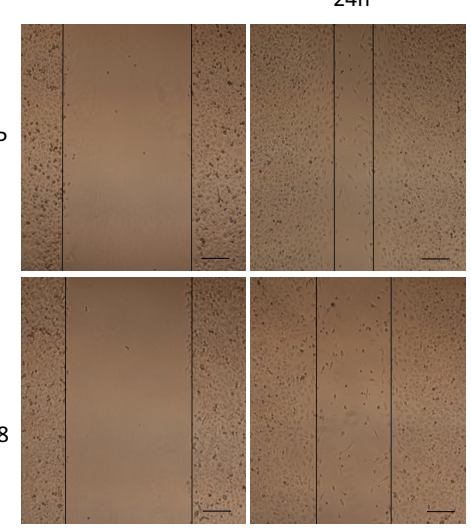

Figure 6 
A

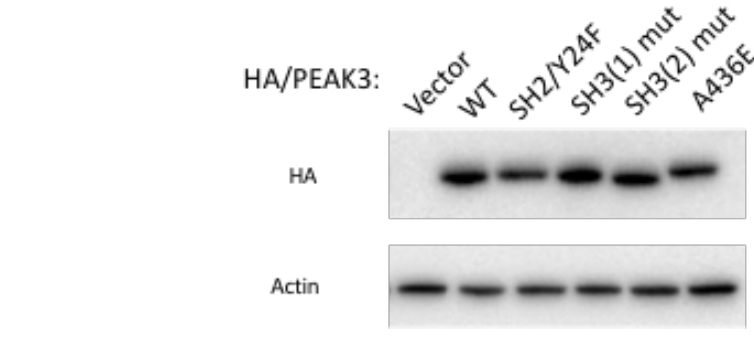

B

C
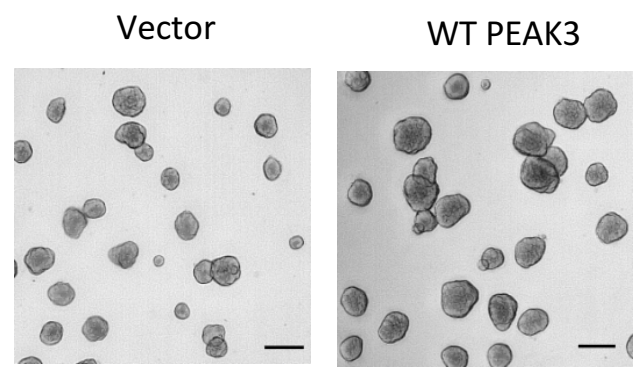

D
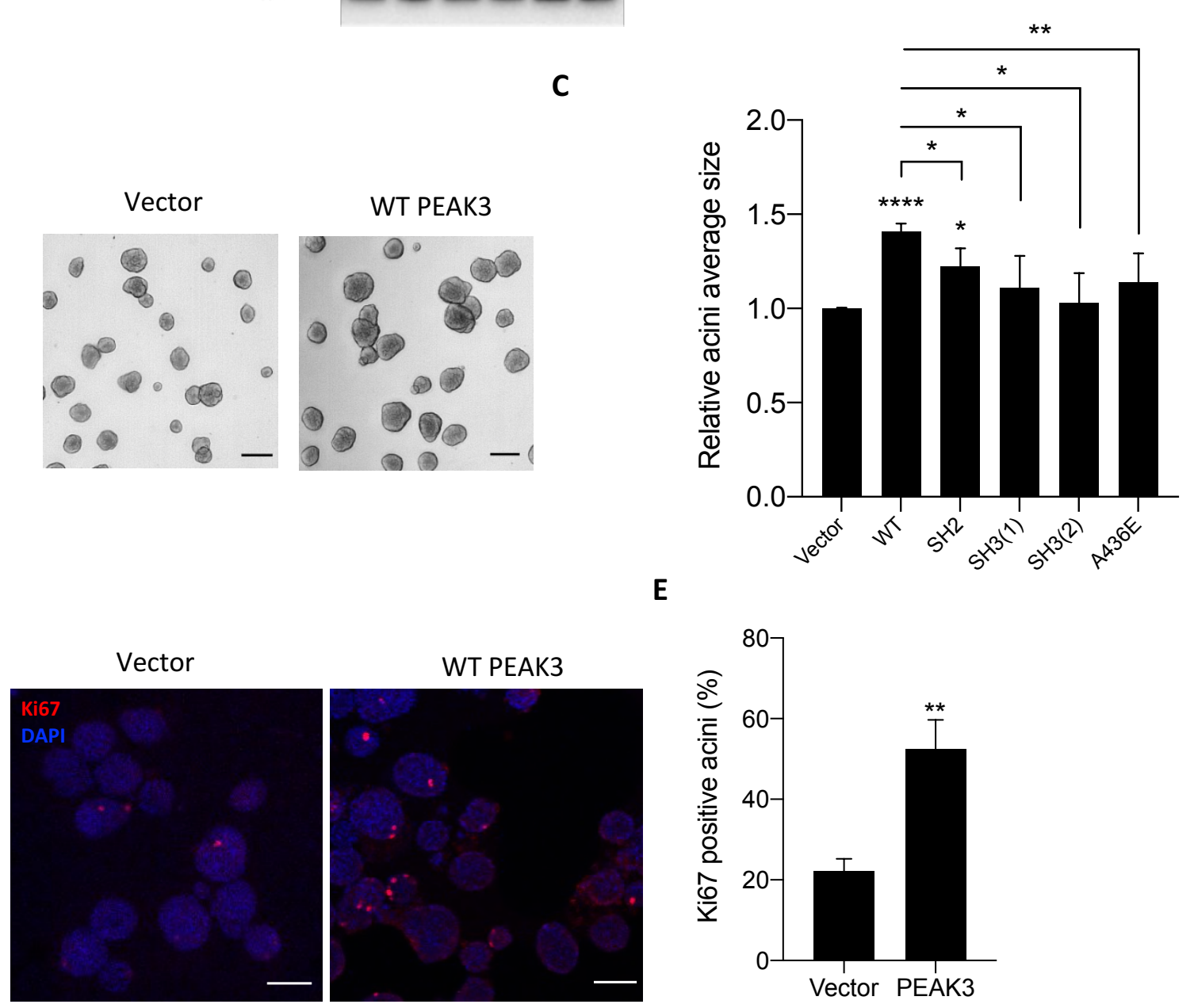

WT PEAK3
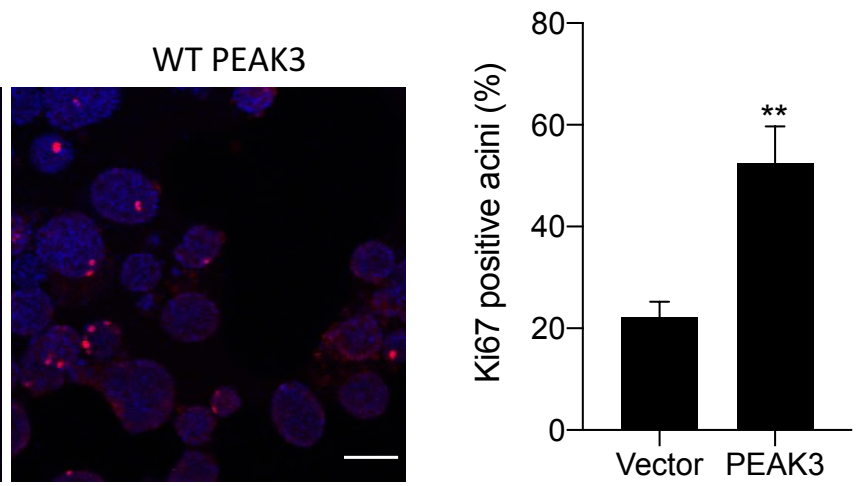

Figure 7 
A
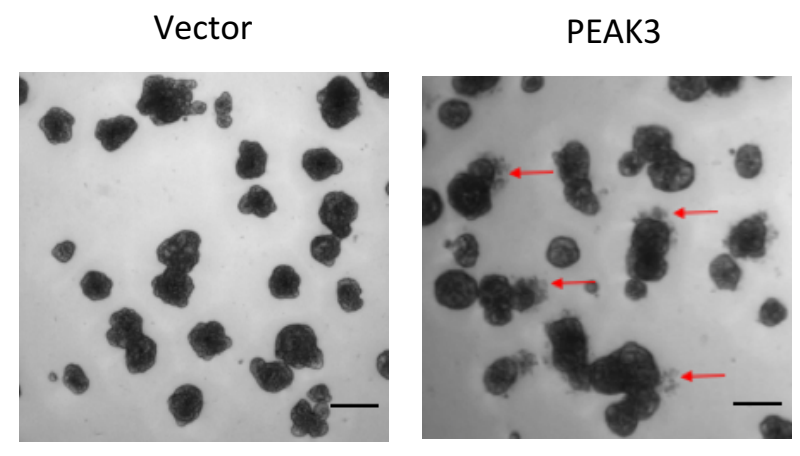

B

Vector
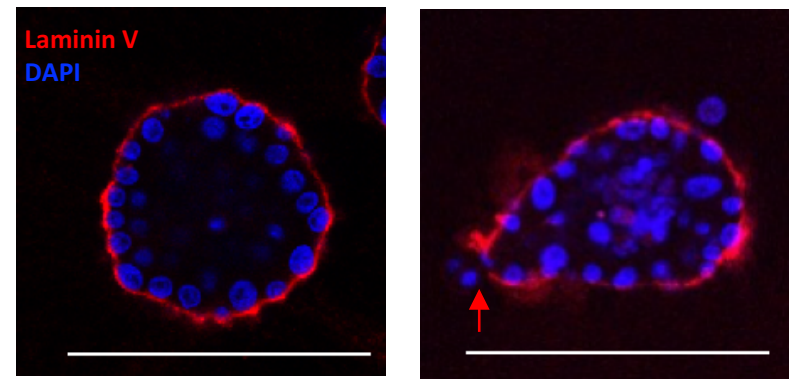

C

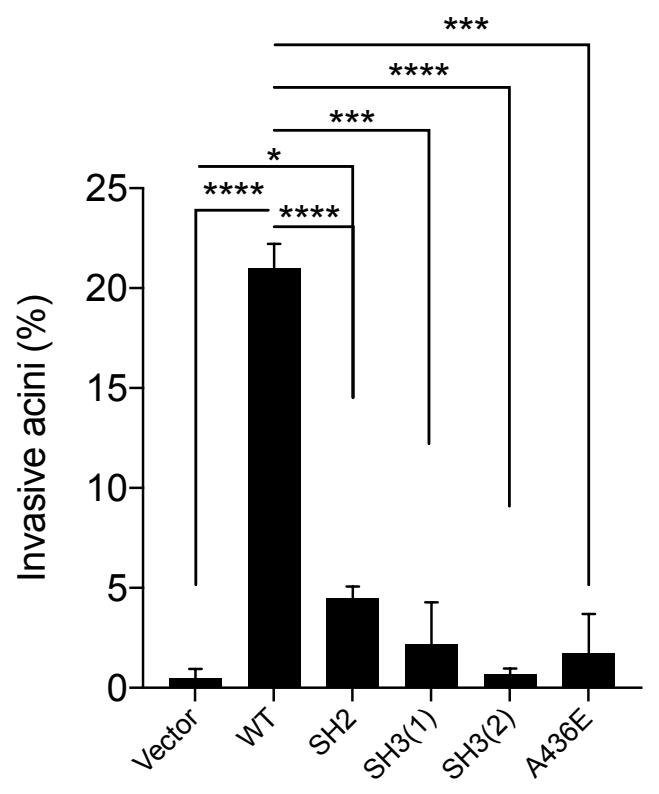

Figure 8 